\title{
State of the Art of Level Set Methods in Segmentation and Registration of Medical Imaging Modalities
}

\author{
Elsa Angelini, Yinpeng Jin, Andrew Laine
}

Columbia University, New York, NY, USA

\begin{abstract}
Segmentation of medical images is an important step in various applications such as visualization, quantitative analysis and image-guided surgery. Numerous segmentation methods have been developed in the past two decades for extraction of organ contours on medical images. Low-level segmentation methods, such as pixel-based clustering, region growing, and filter-based edge detection, require additional pre-processing and post-processing as well as considerable amounts of expert intervention or information of the objects of interest. Furthermore the subsequent analysis of segmented objects is hampered by the primitive, pixel or voxel level representations from those region-based segmentation [1].

Deformable models, on the other hand, provide an explicit representation of the boundary and the shape of the object. They combine several desirable features such as inherent connectivity and smoothness, which counteract noise and boundary irregularities, as well as the ability to incorporate knowledge about the object of interest [2, 3] [4]. However, parametric deformable models have two main limitations. First, in situations where the initial model and desired object boundary differ greatly in size and shape, the model must be re-parameterized dynamically to faithfully recover the object boundary. The second limitation is that it has difficulty dealing with topological adaptation such as splitting or merging model parts, a useful property for recovering either multiple objects or objects with unknown topology. This difficulty is caused by the fact that
\end{abstract}


a new parameterization must be constructed whenever topology change occurs, which requires sophisticated schemes $[5,6]$. Level set deformable models $[7,8]$, also referred to as geometric deformable models, provide an elegant solution to address the primary limitations of parametric deformable models. These methods have drawn a great deal of attention since their introduction in 1988. Advantages of the contour implicit formulation of the deformable model over parametric formulation include: (1) no parameterization of the contour, (2) topological flexibility, (3) good numerical stability, (4) straightforward extension of the 2D formulation to n-D. Recent reviews on the subject include papers from Suri $[9,10]$.

In this chapter we give a general overview of the level set segmentation methods with emphasize on new frameworks recently introduced in the context of medical imaging problems. We then introduce novel approaches that aim at combining segmentation and registration in a level set formulation. Finally we review a selective set of clinical works with detailed validation of the level set methods for several clinical applications.

\section{A. Level set methods for Segmentation}

A recent paper from Montagnat, Delingette and Ayache review the general family of deformable models and surfaces with a classification based on their representation. This classification has been reproduced, to some extends in Figure 1. Level set deformable models appear in this classification diagram as part of continuous deformable models with implicit representation. 


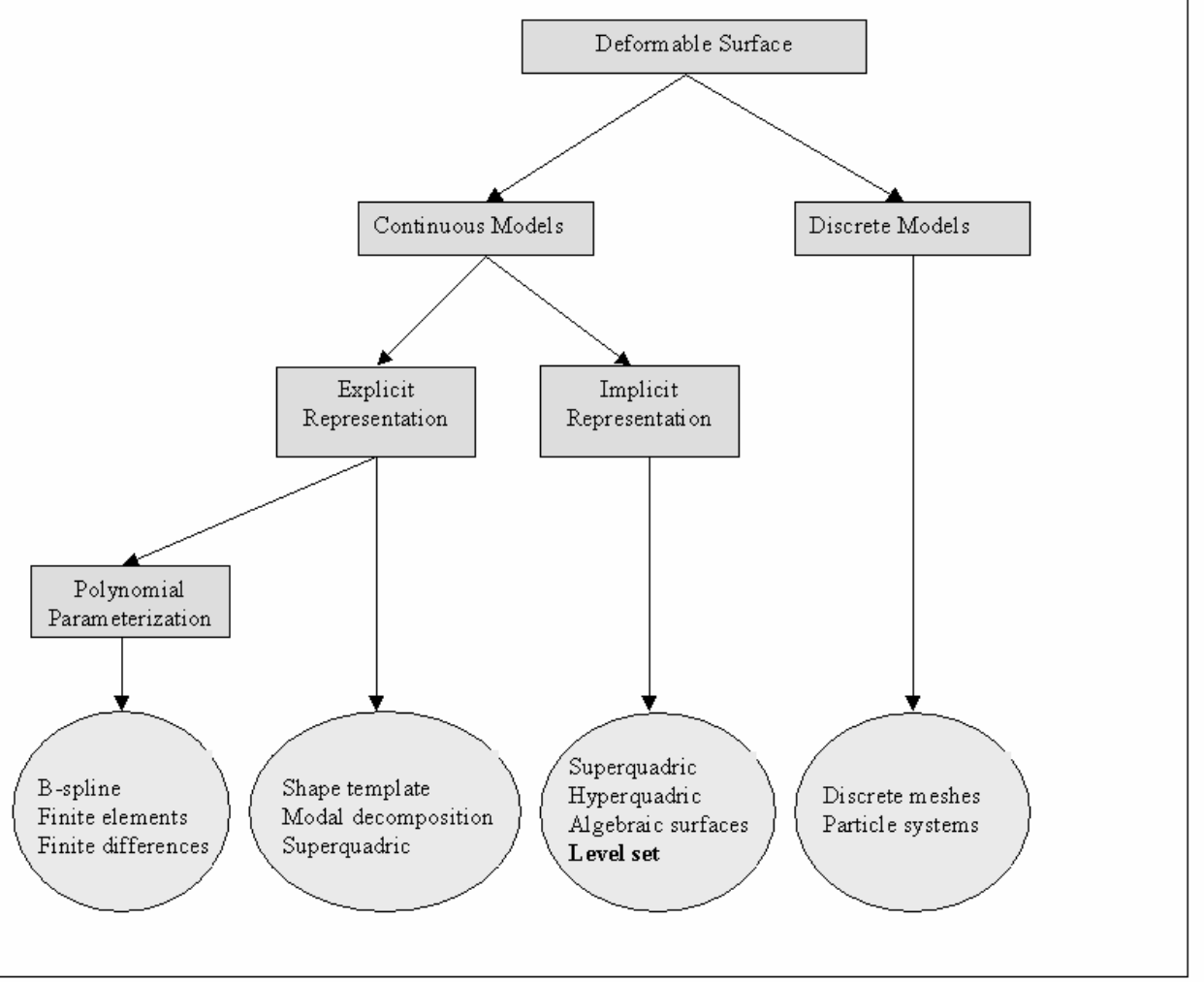

Figure 1:Geometric representations of deformable surfaces.

\section{A. Level Set Framework}

Segmentation of an image $I$ via active contours, also referred to as snakes [2], operates through an energy functional controlling the deformation of an initial contour curve $C(p), p \in[0,1]$ under the influence of internal and external forces achieving a minimum energy state at high-gradient locations. The generic energy functional for active contour models is expressed as:

$$
E(C)=\alpha \int_{0}^{1}\left|C^{\prime}(s)\right|^{2} d s+\beta \int_{0}^{1}\left|C^{\prime \prime}(s)\right| d s-\lambda \int_{0}^{1}|\nabla I(C(s))|^{2} d s
$$


where $(\alpha, \beta, \lambda)$ are positive parameters. The first two terms control the rigidity and elasticity of the contour (defining the internal energy of the deformable object) while the last term attracts the model to high-gradient locations in the image $I$ (defining the external energy of the model).

Active contour segmentation via minimization of the energy functional in Equation (1) is typically implemented with a parametric framework in which the deformable model is explicitly formulated as a parameterized contour on a regular spatial grid, tracking its point positions in a Lagrangian framework [11].

In their original paper from 1988 [7], Osher and Sethian introduced the concept of geometric deformable models, which provide an implicit formulation of the deformable contour in a level set framework. To introduce the concept of the level set framework we focus on the boundaryvalue problem of a close contour $C$ deforming with a speed $V$ along its normal direction:

$$
\begin{aligned}
& |\nabla C| V=1, \\
& V>0
\end{aligned}
$$

Their fundamental idea is, instead of tracking in time the positions of the front $C(x, y)$ on a regular grid as:

$$
\Gamma(t)=\{(x, y) \mid C(x, y)=t\}
$$

to embed the curve into a higher dimension function $\phi(x, y, t)$ such that:

(1) at time zero the initial contour $C_{0}$ corresponds to the level zero of the function $\phi$ :

$$
C_{0}=\{(x, y) / \phi(x, y, 0)=0\}
$$

(2) the function $\phi$ evolves with the dynamic equation:

$$
\frac{\partial \phi}{\partial t}=|\nabla \phi| V
$$

In this framework, at any time $t$, the front implicitly defined by:

$$
\Gamma(t)=\{(x, y) / \phi(x, y, t)=0\}
$$


corresponds to the solution of the initial boundary value problem defined parametrically in Equation (3). This result is illustrated in Figure 2.

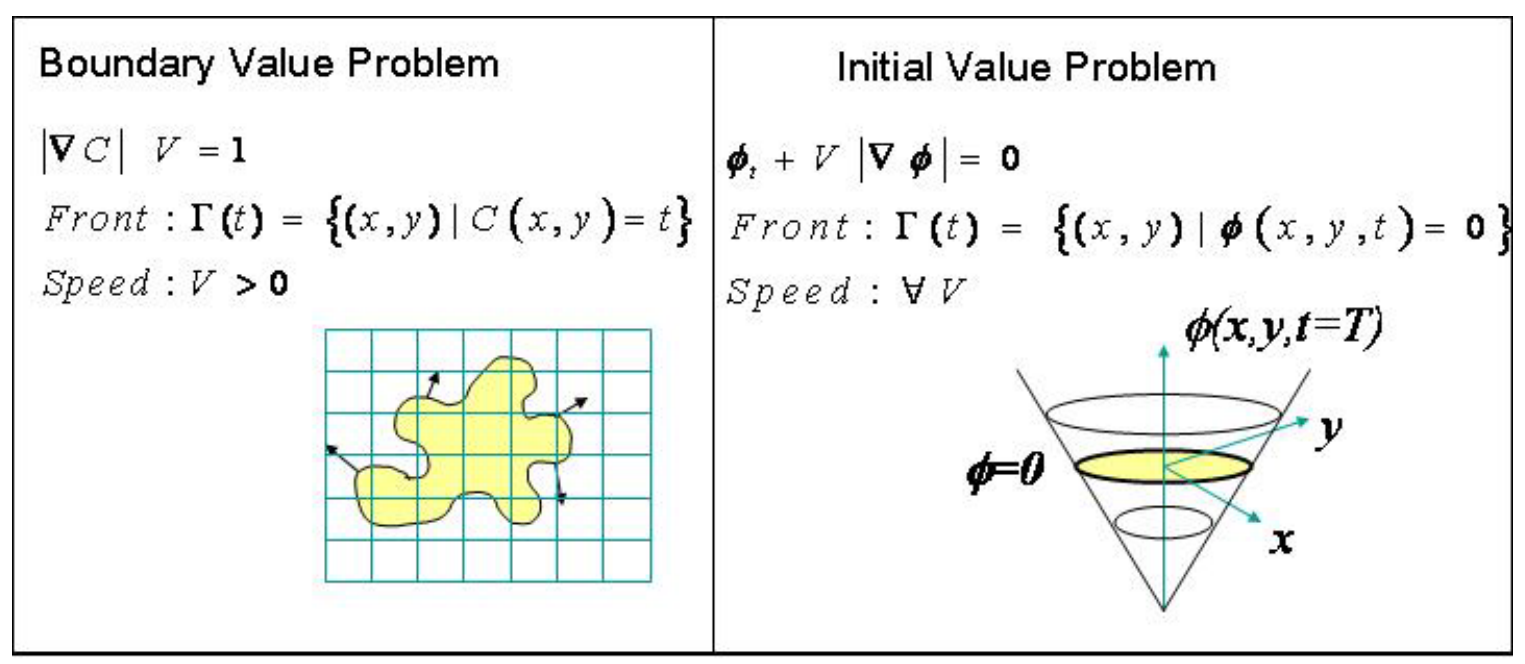

Figure 2: Correspondence between a parametric and implicit level-set formulation of the deformation of a contour with a speed term oriented along the normal direction.

In their pioneer paper, Osher and Sethian focused on motion under mean curvature flow where the speed term is expressed as:

$$
V=\operatorname{div}\left(\frac{\nabla \phi}{|\nabla \phi|}\right)
$$

Since its introduction, the concept of deformable models for image segmentation defined in a level set framework has motivated the development of several families of method that include: geometric active contours based on mean curvature flow, gradient-based implicit active contours and geodesic active contours.

\section{B. Geometric Active Contours}

In their work introducing geometric active contours, Caselles et al. [12] proposed the following functional to segment a given image $I$ : 


$$
\frac{\partial \phi}{\partial t}=|\nabla \phi| g(|\nabla I|)\left(\operatorname{div}\left(\frac{\nabla \phi}{|\nabla \phi|}\right)+\nu\right)
$$

with

$$
g(|\nabla I(x, y)|)=\frac{1}{1+\left|\nabla G_{\sigma}(x, y) * I(x, y)\right|^{2}},
$$

where $\nu \geq 0$ and $G_{\sigma}$ is a Gaussian convolution filter of standard deviation $\sigma$. The idea defining geometric deformable models is to modify the initial mean curvature flow of Equation (7) by adding a constant inflation force term $\nu$ and multiplying the speed by a term inversely proportional to the smooth gradient of the image. In this context the model is forced to inflate on smooth areas and to stop at high-gradient locations as the speed decreases towards zero.

\section{Gradient-Based Level Set Active Contours}

In their initial work on applications of the level set framework for segmentation of medical images, Malladi, Sethian and Vemuri [8] presented a gradient-based speed function for the general Hamilton-Jacobi type equation of motion in Equation (5).

Their general framework decomposed the speed term into two components:

$$
V=V_{a}+V_{G}
$$

where $V_{a}$ is an advection term, independent of the geometry of the front and $V_{G}$ is a remainder term that depends on the front geometry.

The authors studied the design of the speed term to stop the front propagation at high-gradient locations depending on the value of $V_{G}$.

In the first case, for $V_{G}=0$ they proposed the following speed term:

$$
V=\left(-V_{a}+\frac{V_{a}}{\left(M_{1}-M_{2}\right)}\left(\left|\nabla G_{\sigma} * I\right|-M_{2}\right)\right),
$$


where $\left(M_{1}, M_{2}\right)$ are the maximum and minimum values of the smooth gradient image $\left|\nabla G_{o}^{*} I\right|$

In the case where $V_{G} \neq 0$, the speed term needs to be multiplied by a gradient-based term to stop the front evolution, as follows:

$$
V=\left(\frac{1}{1+\left|\nabla G_{\sigma}^{*} I\right|}\right) \times\left(V_{a}+V_{G}\right) .
$$

Numerical schemes for approximation of spatial derivatives with theses speed terms must respect the appropriate entropy condition for propagating fronts as discussed in detailed in [13] and [14]. This entropy condition ensures that the propagating front corresponds to the boundary of an expanding region. An analogy invoked by Sethian to illustrates the entropy principle is to consider the moving front as a source for a burning flame and expand the flame so ensuring that once a point in the domain is ignited, it stays burnt. The entropy principle puts some constraints in the choice of particular numerical schemes for temporal and spatial derivatives of the level set function. In their work, Malladi et al. [8] used a forward difference in time, upwind scheme for the constant inflation term and central differences for the remainder term.

A second issue with this framework arises from the fact the image-based speed terms are only defined on the zero-level of the moving front, as it was designed to stop the evolution of this level at high-gradient locations. On the other hand the energy functional is defined over the entire domain and the speed term must have a consistent definition over all values of the level set function. This is done by extending the speed term from its values defined only on the level zero. There are several methods available to perform the extension. One of the most popular methods assigns the values of the closest point on the level zero to a given point of the domain. 


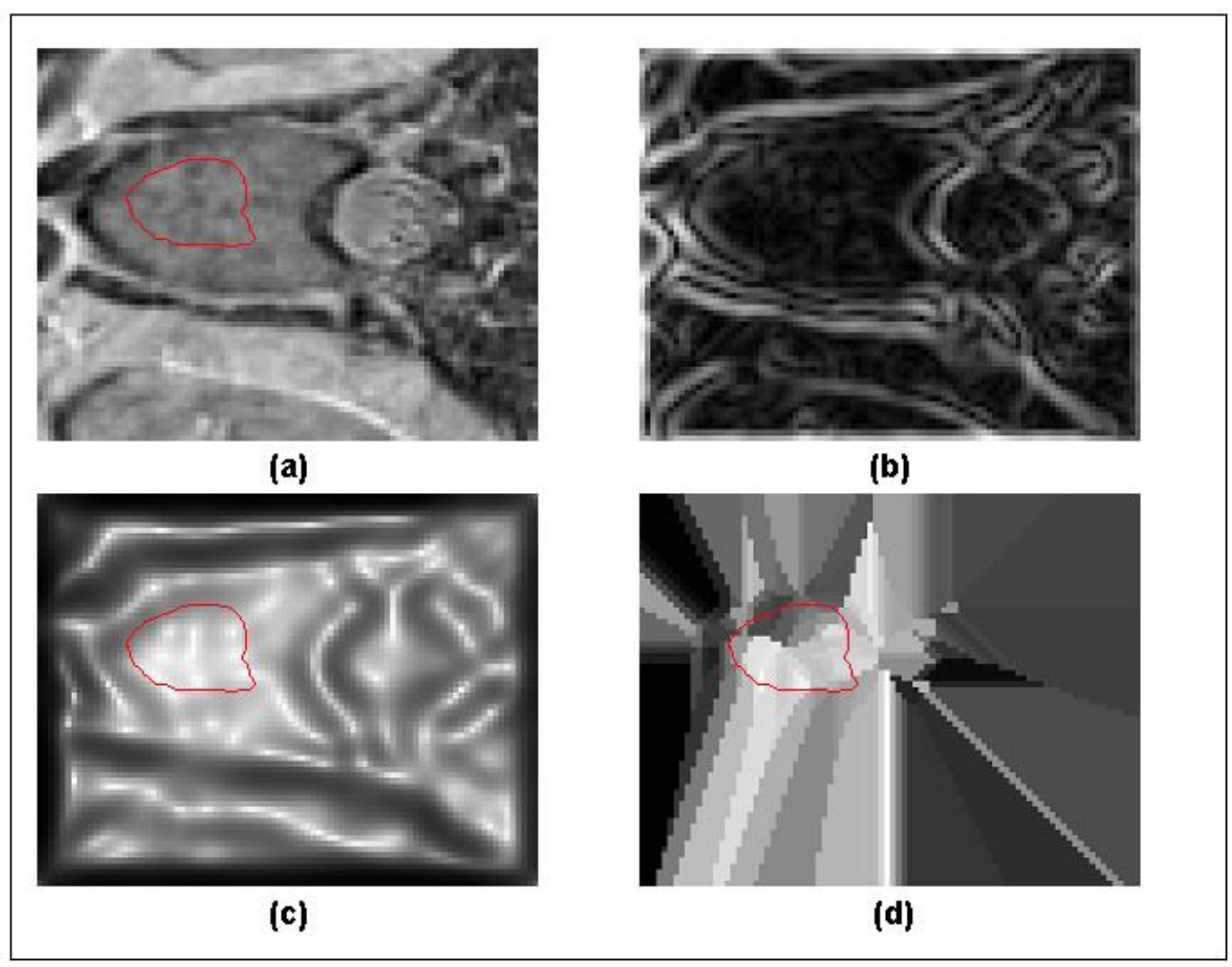

Figure 3: Illustration of extension for gradient-based speed terms. (a) Region of interest from a chest MRI scan with a level set curve initialized inside the spine. (b) Gradient map of the MRI image with prior smoothing with a Gaussian filter. (c) Multiplying term for speed function, proportional to the inverse edge map from (b). (d) Extension of the speed term in (c) from values under the zero level contour.

\section{Geodesic Active Contours}

Geodesic active contours were introduced simultaneously by Kichenassamy and al. [15] and Caselles et al. [16] as a segmentation framework, derived from energy-based snakes active contours, performing contour extraction via the computation of geodesics, i.e. minimal distance curves in a Riemannian space derived from the image. Given an image $I$ and for a given differentiable curve $C(p), p \in[0,1]$ they define the following energy: 


$$
E(C)=\int_{0}^{1} g(|\nabla I(C(p))|)\left|C^{\prime}(p)\right| d p
$$

where $g$ is a positive decreasing function. Segmentation is achieved via minimization of this energy functional equivalent to the computation of geodesics in a Riemannian space according to a metric that weights the Euclidian length of the curve with the term $g(|\nabla I(C(p))|)$.

Minimization of the functional is performed via derivation of the Euler-Lagrange system:

$$
\frac{\partial C}{\partial t}=g(|\nabla I|) \kappa \vec{N}-(\nabla g(|\nabla I|) \cdot \vec{N}) \vec{N}
$$

where $\kappa$ is the Euclidian curvature of the curve $C$ and $\vec{N}$ is the unit normal vector to the curve. Implementation with a level set framework is performed by embedding the curve $C$ into a level set function $\phi$. Using the following property on the curvature term:

$$
\kappa=\operatorname{div}\left(\frac{\nabla \phi}{|\nabla \phi|}\right),
$$

and the following equivalence of relationships between a curve $C$ and its associated level set function $\phi$ :

$$
\begin{aligned}
& \frac{\partial C}{\partial t}=\alpha \vec{N} \\
& \frac{\partial \phi}{\partial t}=\alpha|\nabla \phi|
\end{aligned}
$$

the level set formulation is expressed as: 
To improve convergence speed and allow the detection of non-convex objects, the authors also introduced a modification of the initial formulation with the introduction of a constant inflation term $\nu g(|\nabla I|)|\nabla \phi|$ leading to the following functional:

$$
\begin{aligned}
\frac{\partial \phi}{\partial t} & =|\nabla \phi|\left(\operatorname{div}\left(g(|\nabla I|) \frac{\nabla \phi}{|\nabla \phi|}\right)+\nu g(|\nabla I|)\right) \\
& =g(|\nabla I|)(\kappa+\nu)|\nabla \phi|+\nabla g(|\nabla I|) \nabla \phi
\end{aligned}
$$

Applications of the geodesic deformable model to medical imaging have been tested by both groups of pioneering authors. Yezzi et al. tested their geodesic deformable model in [17] on 2D images for cardiac MRI, breast ultrasound with a cyst and bone CT. No clinical validation was performed. Caselles, with Malladi et al. in [18] compared 2D geometric and 3D geodesic deformable models with applications on 3D CT of human thighs and 3D cardiac MRI data sets. Measurement of soft tissue and ventricular cavity volumes are reported but no clinical validation was performed. A recent review of the use of geodesic deformable models for medical image analysis is provided in [19] with comparison of performance between geometric and parametric deformable models.

\section{E. Tuning Level Set Speed Functions for Segmentation}

The main problem of boundary-based level set segmentation methods is related to contour leakage at locations of weak or missing boundary data information. An illustration of the phenomenon is provided in Figure 4 for segmentation of a high-resolution abdominal MRI slice. 


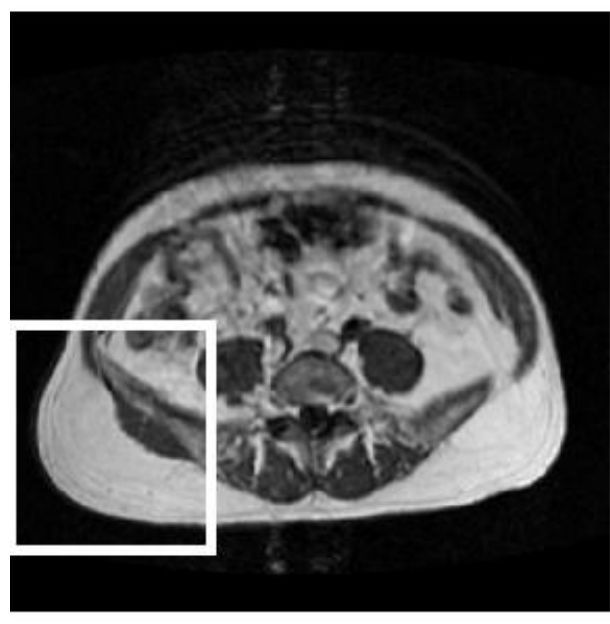

(a)

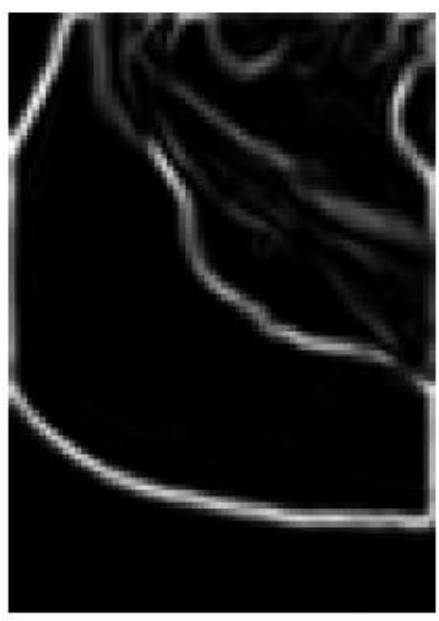

(b)

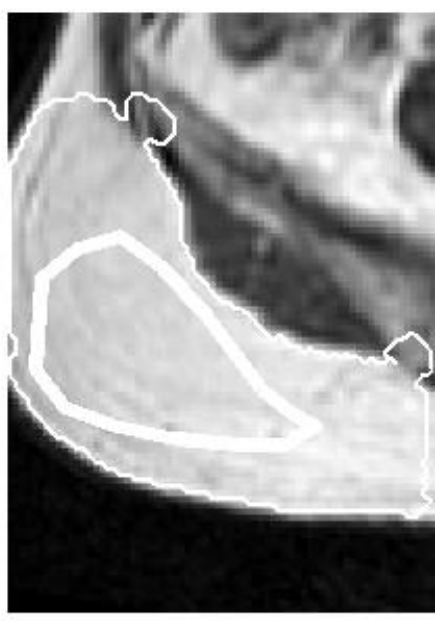

(c)

Figure 4: Leakage of level set deformable model at location of weak edges with gradient-based speed terms. (a) T2-weighted abdominal MRI with region of interest selected to contain sub-cutaneous fat. (b) Edge map derived from gradient computation to define speed term. (c) Leakage of front outside the fat compartment at two locations due to interstices with poor edge contrast. The level zero curve used to initialize the segmentation is displayed with a thick line.

Several efforts have been performed to add stopping criteria on the entire front $[20,21]$ and local pixel freezing rules [21], or combine gradient with region information [22, 23] to make the segmentation process more robust to poor edge definition. When dealing with weak boundaries the most radical solution to leaking problems is to remove the expansion term at the cost of requiring an initialization close to the final solution [24]. An alternative to this approach was proposed by Jin et al. [25] initially keeping the expansion term for pushing the model and turning it off as it approaches the object boundary. Detection of the boundary location was performed using a homogeneity map derived from scale-based fuzzy connectivity [26]. A more recent effort to address the problem of segmentation of an object with missing boundaries was presented by Sarti, Malladi and Sethian in [27] introducing a new geometric model for subjective surfaces. Starting from a reference point inside the object to segment, the 'point of view', a geometric 
deformable model is evolved with mean curvature flow and image-derived speed terms until a piecewise constant solution is reached. This piecewise constant solution is the subjective surface defined by the segmentation process that is flat inside the object and has boundaries defined by geodesic curves. The authors also introduced the notion of "modal" contours which are contours that are perceived in the visual context and "amodal" contours which are associated with partially occluded objects. Segmentation of amodal contours can be performed with their subjective surface framework through iterations of edge-map computation and contour extraction. The authors produced very nice illustrations of the performance of their subjective surface segmentation on three-dimensional ultrasound data with a fetal echogram, recovering the shape of the fetus.

All the level set segmentation methods presented above are based on image gradient intensity making them prone to leaking problems in areas with low contrast. A second problem related to the use of the image gradient as the only image-derived speed term is that it makes the segmentation process very sensitive to the initial position of the level set function as the model is prone to converge to false edges that correspond to local minima of the functional. Medical images typically suffer from insufficient and spurious edges inherent to physics of acquisition and machine noise from different modalities.

Two approaches can be followed to address these limitations. The first approach is to fuse regularizer terms in the speed function as reviewed in [9]. A second approach is to reformulate the problem in terms of region-based segmentation methods derived from the Mumford-Shah functional implemented in a level set framework. We give an overview of these two families of methods in the next section.

\section{F. Level Set Speed Functions with Regularizers}

Suri et al. review in [9] recent works on the fusion of classical geometric and geodesic deformable models speed terms with regularizers, i.e. regional statistics information from the 
image. Regularization of the level set speed term is desirable to add prior information on the object to segment and prevent segmentation errors when using only gradient-based information in the definition of the speed. Four main types of regularizers were identified by the authors of the review:

(1) Clustering-based regularizers.

(2) Bayesian-based regularizers.

(3) Shape-based regularizers.

(4) Coupling-surfaces regularizers.

We give in the next cession a brief overview of each method.

(1) Clustering-Based Regularizers: Suri proposed in [28] the following energy functional for level set segmentation:

$$
\frac{\partial \phi}{\partial t}=\left(\varepsilon \kappa+V_{p}\right)|\nabla \phi|-V_{e x t} \nabla \phi
$$

where $V_{p}$ is a regional force term expressed as a combination of the inside and outside regional area of the propagating curve. This term is proportional to a region indicator taking value between 0 and 1, derived from a fuzzy membership measure as described in [29].

(2) Bayesian-Based Regularizers: Recent work from Baillard et al. [30] proposed an approach similar to the previous one where the level set energy functional expressed as:

$$
\frac{\partial \phi}{\partial t}=g(|\nabla I|)\left(\kappa+V_{0}\right)|\nabla \phi|
$$

uses a modified propagation term $V_{0}$ as a local force term. This term was derived from the probability density functions inside and outside the structure to segment. The authors also modified the data consistency term $g(|\nabla I|)$ as expressed in Equation (9) using a transitional probability from going inside to outside the object to be segmented. 
(3) Shape-Based Regularizers: Leventon et al. [31] introduced shape-based regularizers where curvature profiles act as boundary regularization terms more specific to the shape to extract than standard curvature terms. A shape model is built from a set of segmented exemplars using principle component analysis applied to the signed-distance level set functions derived from the training shapes. The principal modes of variation around a mean shape are computed. Projection coefficients of a shape on the identified principal vectors are referred to as shape parameters. Rigid transformation parameters aligning the evolving curve and the shape model are referred to as pose parameters. To be able to include a global shape constraint in the level set speed term, shape and pose parameters of the final curve $\phi^{*}(t)$ are estimated using maximum a posteriori estimation. The new functional is derived with a geodesic formulation as in Equation (18) with solution for the evolving surface expressed as:

$$
\phi(t+1)=\phi(t)+\lambda_{1}(g(|\nabla I|)(c+\kappa)|\nabla \phi(t)|+\nabla g(|\nabla I|) \cdot \nabla \phi(t))+\lambda_{2}\left(\phi^{*}(t)-\phi(t)\right),
$$

where $\left(\lambda_{1}, \lambda_{2}\right)$ are two parameters that balance the influence of the gradient-curvature term and the shape-model term. In more recent work, Leventon at al. [32] introduced further refinements of their method by introducing prior intensity and curvature models using statistical image-surface relationships in the regularizer terms. Limited clinical validation have been reported using this method but some illustrations on various applications including segmentation of the femur bone, the corpus callosum and vertebral bodies of the spine showed efficient and robust performance of the method.

(4) Coupling-Surfaces Regularizers: Segmentation of embedded organs such as the cortical gray matter in the brain have motivated the introduction of a level set segmentation framework to perform simultaneous segmentation of the inner and outer organ surfaces with coupled level set functions. Such method was proposed by Zeng et al. in [33]. In this framework, segmentation is performed with the following system of equations: 


$$
\left\{\begin{array}{l}
\phi_{\text {in }}+V_{\text {in }}\left|\nabla \phi_{\text {in }}\right|=0 \\
\phi_{\text {out }}+V_{\text {out }}\left|\nabla \phi_{\text {out }}\right|=0
\end{array}\right.
$$

where the speed terms $\left(V_{\text {in }}, V_{\text {out }}\right)$ are functions of the surface normal direction (e.g. curvature of the surface), image-derived information and the distance between the two surfaces. When this distance is within the desired range, the two surfaces propagate according to the first two terms of the speed term. When the distance is out of the desired range, the speed term based on the distance controls the deformation as to correct for the surface positions.

When defining the initial level set function as the signed distance function to its level zero, and ensuring that the distance function is preserved during the deformation process of the front through reinitialization, the distance of any point on the inner surface to the outer surface is directly read as the value of the outer level set function and vice versa.

Defining the speed terms as:

$$
\left\{\begin{array}{l}
V_{\text {in }}=F_{\text {in }} h\left(\phi_{\text {out }}\right) \\
V_{\text {out }}=F_{\text {out }} h\left(\phi_{\text {in }}\right)
\end{array}\right.
$$

with $\left(F_{\text {in }}, F_{\text {out }}\right)$ speed terms derived from image and curvature properties and $h()$ a smooth approximating the windowing step function defined for a range of distance $\left[d_{1} d_{2}\right]$ that is equal to one inside this interval and 0 outside.

Zeng et al. [33] applied this framework for the segmentation of brain cortical gray matter (GM) surfaces. In this application, the speed terms were defined as:

$$
\left\{\begin{array}{l}
V_{\text {inside }}=S^{-}\left(I-I_{\text {in }}\right)+S^{+}\left(\phi_{\text {out }}+\varepsilon\right) \\
V_{\text {outside }}=S^{-}\left(I-I_{\text {out }}\right)+S^{+}\left(\phi_{\text {in }}-\varepsilon\right)
\end{array}\right.
$$

where $I$ is the intensity of the MRI, $I_{i n}$ is a threshold value corresponding to the white matter and $I_{\text {out }}$ a threshold value corresponding to the gray matter, $\varepsilon$ is the desired thickness of the gray 
matter layer, $\left(S^{-}, S^{+}\right)$are two sigmoid functions respectively decreasing and increasing with bounded value between $[-1,1]$. If the curve evolution is implemented with Equation (5), the magnitude of the gradients $\left(\left|\nabla \phi_{\text {in }}\right|,\left|\nabla \phi_{\text {out }}\right|\right)$ will increase and the estimation of the distance between the zero-levels of the two functions will be overestimated, leading $\left(\phi_{\text {in }}, \phi_{\text {out }}\right)$ to get closer as they evolve and eventually collide until the level set functions are reinitialized. Results are illustrated on three regions of interest from three MRI slices and show very interesting results but no quantitative evaluation of the accuracy of the method was performed.

\section{G. Reconciling Level Set and Distance Function}

In a recent paper [34], Gomes and Faugeras introduced a reformulation of the Hamilton-Jacobi equation of Equation (5) underlying the level set initial formulation from Osher and Sethian [7] to eliminate problems related to reinitialization of the distance function and the need to extend the velocity field away from the level zero.

The fact that the solution to Hamilton-Jacobi equations of the form in Equation (5) are not distance functions has been demonstrated formally in [35]. The authors is [34] provide two simple examples illustrating this result. There are both theoretical and practical reasons pointed out by the authors to motivate the preservation of the signed distance function during the segmentation process. Theoretically, the signed distance function gives a unique equivalence to the implicit description of the moving front. From a practical point of view, the use of a signed distance function enables to directly extract from the level set function geometrical properties of the front and guarantees bounded values of the level set function gradient, ensuring numerical stability of the segmentation iterative process.

To derive the new dynamic equation, the authors initialize the level set function $\phi_{0}=\phi(x, 0)$ 
at $\mathrm{t}=0$ as the signed distance function from the initial front. The goal is to redefine a speed function $\mathrm{F}$ such that $\frac{\partial \phi}{\partial t}=F$ which (1) preserves $\phi$ as the signed distance function from the level zero, and (2) ensures that the level zero of $\phi$ evolves as in Equation (2). These constraints are expressed mathematically as:

$$
\left\{\begin{array}{l}
F_{/ \phi=0}=V \\
\frac{\partial \phi}{\partial t}=F \\
|\nabla \phi|=1
\end{array}\right.
$$

where $F_{/ \phi=0}$ denotes the restriction of $F$ to the zero-level of $\phi$. The authors derived the following dynamic equation as the solution to this system:

$$
\frac{\partial \phi}{\partial t}=V(x-\phi \nabla \phi)
$$

for any point $x \in \mathbb{R}^{3}$, which is not a Hamilton-Jacobi equation.

Implementation of the equation is proposed with a narrow-band framework, shock-detecting gradient computation and as described in [14].

The authors provide a very nice application for segmentation of cortical gray matter surfaces from MRIs derived from the initial work of Zeng et al. [33]. With this method, if the curve evolution in Equation (24) is implemented with model in Equation (5), the magnitude of the gradients $\left(\left|\nabla \phi_{\text {in }}\right|,\left|\nabla \phi_{\text {out }}\right|\right)$ will increase and the estimation of the distance between the zero-levels of the two functions will be overestimated, leading $\left(\phi_{\text {in }}, \phi_{\text {out }}\right)$ to get closer as they evolve and eventually collide until the level set functions are reinitialized. Results are illustrated on three regions of interest from three MRI slices and show very interesting results but no quantitative evaluation of the accuracy of the method was performed. 


\section{H. Region-Based Level Set Active Contours}

Region-based active contour were derived from the Mumford-Shah segmentation framework initially proposed in [36]. In their initial work, Mumford and Shah defined a new segmentation framework performing segmentation of a given image $I$ into a set of contours $S$ and a smooth approximation $f$ of the image via minimization of the following framework:

$$
E(S, f)=\alpha \int_{\Omega}(f-I)^{2} d x+\beta \int_{\Omega \backslash S}|\nabla f| d x+H^{n-1}(S)
$$

where $H^{n-1}(S)$ is the $(n-1)$ dimensional Hausdorff measure, and $(\alpha, \beta)$ are positive real parameters. In this functional, the first term ensures that $f$ is a good approximation of the original image $I$, the second term ensures that $f$ is smooth and the last term minimizes the length of the set of contours of the segmentation. This type of region-based segmentation method relies on the homogeneity of the object to segment. This assumption is often violated with medical images due to motion of the organ, presence of corrupting machine noise or acquisition artifacts that introduce flat field inhomogeneities.

Based on the Mumford-Shah segmentation framework, Chan and Vese introduced in a series of papers a new type of active contour models without gradient information [37-41]. In the simplest case, assume that an image $I$ defined on $\Omega$ is composed of two regions (e.g. an object and a background) with homogeneous intensities around values $C_{0}$ and $C_{1}$. Given a curve $C$ that defines the boundary of a region inside the image $I$, they introduce the following homogeneity-based functional:

$$
E(C)=\int_{\text {insideC }}\left|I-C_{0}\right|^{2} d \Omega+\int_{\text {outsideC }}\left|I-C_{1}\right|^{2} d \Omega
$$

where $\left(C_{0}, C_{1}\right)$ are the average intensity values of $I$ inside and outside the curve $C$. With this functional the boundary between the two regions is defined by its minimum state. They further 
combined this homogeneity-based fitting term with regularizing terms that put constraints on the length and the area of the curve with the following functional:

$$
E\left(C_{0}, C_{1}, C\right)=\lambda_{0} \int_{\text {insideC }}\left|I-C_{0}\right|^{2} d \Omega+\lambda 1 \int_{\text {outsideC }}\left|I-C_{1}\right|^{2} d \Omega+\mu \text { length }(C)+\mu \operatorname{Area}(C) .
$$

Details for the mathematical definitions of the length and the area of the boundary curve $C$ can be found in [40].

In a level-set framework implementation, the functional (29) is expressed as:

$$
\begin{aligned}
E\left(C_{0}, C_{1}, \phi\right)= & \lambda_{0} \int\left|I-C_{0}\right|^{2} H(\phi) d \Omega+\lambda_{1} \int\left|I-C_{1}\right|^{2}(1-H(\phi)) d \Omega+ \\
& \mu \int \delta(\phi)|\nabla \phi| d \Omega+\nu \int H(\phi) d \Omega
\end{aligned}
$$

Advantages of this method include the possibility of segmenting objects with discontinuous edges and robustness of the method to arbitrary initialization, avoiding the problem of local minima at spurious edge locations or leakage of the model at missing edge locations. The initial work from these authors have generated many applicative research works for segmentation of medical images, starting with works from the authors themselves in [37] with illustration of their method on brain MRI. three-dimensional ultrasound

A simultaneous and parallel effort to the work of Chan and Vese, from Tsai, Yezzi et al. [42] proposed a reformulation of the Mumford-Shah functional from a curve evolution perspective using a gradient flow formulation and a level set framework implementation. Recent works applying this segmentation method to three-dimensional cardiac ultrasound include Angelini et al. [43], and Lin et al. [44].

We note two powerful extensions of this region-based implicit deformable model for applications to medical images: 
(1) This method is easily extended to segmentation of vectorial images with integration of the multiple channels information in the homogeneity measure. This property, described in details in [45], has potential applications in segmentation of multi-protocols MRI brain data sets or any coregistered multi-modality data sets where combination of spatial information can assist the definition of a particular organ contours.

(2) This method is extensible to multi-phases segmentation using a system of $n$ coupled dynamic PDEs with $\left\{\phi_{1}, \ldots, \phi_{n}\right\}$ level set functions defining $2^{n}$ phases in the segmented data. Extensive description of the multi-phase method is provided in [41]. Potential applications of the multiphase formulation include again segmentation of brain MRIs into multiple tissue types. An illustration of this application is provided in

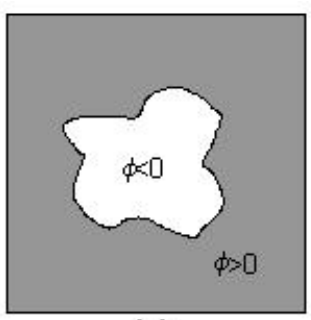

(a)

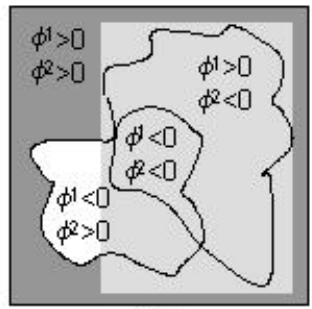

(b)

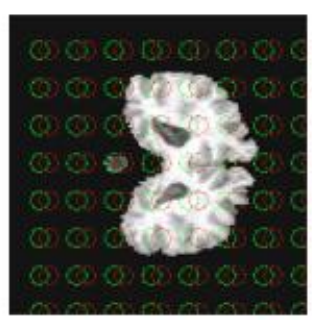

(c)
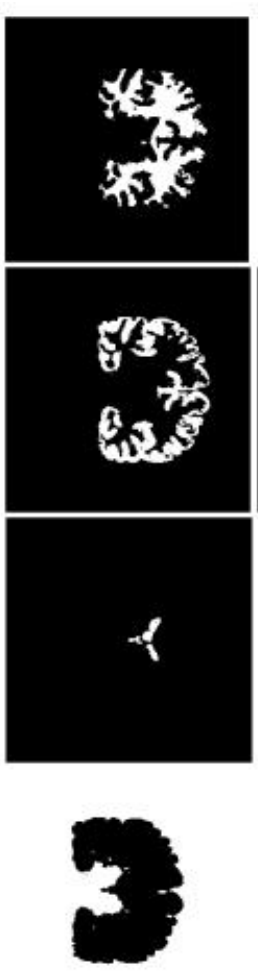

(d)
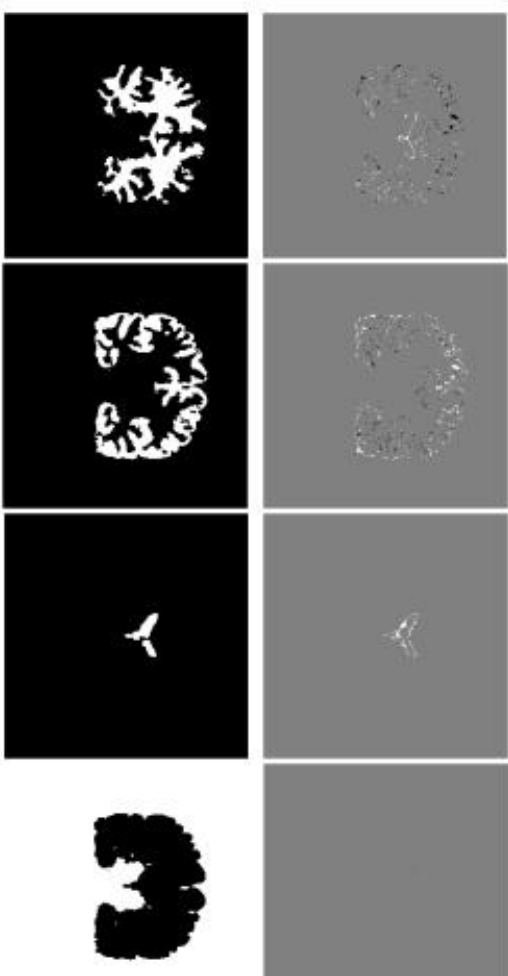

(e)

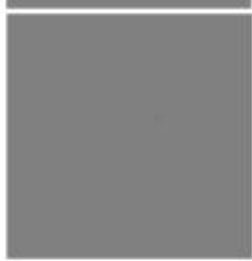

(f)

Figure 5: Multi-phase segmentation of brain MRI with region-based implicit deformable model. (a) Diagram illustrating the definition of two phases with one level set function. (b) Diagram illustrating 
the definition of 4 phases with 2 level set functions. (c) Original axial slice from 3D brain MRI data set with initialization of the two level set functions. (d) Results of implicit deformable model for from each phase: WM (1rst line), GM $\left(2^{\text {nd }}\right.$ line), CSF $\left(3^{\text {rd }}\right.$ line) and background $\left(4^{\text {th }}\right.$ line). (e) Manually labeled data. (f) TP/FP error maps.

\section{B. Joint Image Registration and Segmentation}

\section{A. Motivations}

Combining registration and segmentation has been motivated by the need to incorporate prior information to guide and constrain the segmentation process. The quality of the images acquired by the various medical screening modalities is often poor due to the presence of multiple noise sources in the acquisition system, degradation of data content during reconstruction processes (e.g. tomographic reconstruction with Radon transform), motion and respiratory artifacts introduced by motion of the patient, and inherent limitations of system acquisition accuracy. The combination of these factors degrade the signal to noise ratio of the data, limit the spatial resolution, introduce inhomogeneities in the tissue appearance across volumetric slices, and deteriorate boundary definitions between specific organs and their surrounding tissues. These issues are encountered with other medical imaging modalities such as ultrasound, MRI, PET and SPECT and CT.

In the context of brain MRI segmentation for example, incorporation of atlas information to assist the segmentation task of a particular data set has been a very successful and popular approach for many years as reviewed in [46]. For organs with very characteristics shapes such as cardiac ventricles, the corpus callosum in the brain, or cartilages of the knee, shape priors (including active shape models, active appearance models and statistical shape descriptors) have been used with great success in the context of constrained segmentation [47-50]. 
The use of an atlas (or a shape model) to assist the segmentation process requires that the target image data and the atlas (or the model) are being aligned via either pre-registration or via a new concept of combined registration and segmentation. When considering registration as a preprocessing step, common atlas-based segmentation methods use warping of the atlas to the target data via minimization of mean square errors of image pair intensities at control points. Alternative popular techniques use robust estimators, optimization of correlation ratios, optical flow, fluid-flow non-rigid deformation models and mutual information methods to construct statistical deformation models. A extensive review of registration methods applied to medical imaging can be found in [51]. Among recent work in this area we mention here the method of Vemuri et al. [52] who derived a novel curve evolution approach in a level set framework for image intensity morphing and non-linear associated PDE for the corresponding coordinate registration between an atlas and an image. Applications of the method included a clinical study on segmentation of the corpus callosum via morphing of a shape model defined in the atlas space, after registration of the data with the proposed method.

We focus in this chapter on methods that explicitly combine segmentation and registration in a variational framework. By combining registration and segmentation, one can recover the image region that corresponds to the organ of interest, given a model of this structure. Level set deformable models offer a very flexible framework to propagate a moving front with segmentation-driven constraints while registering the segmentation result (i.e. the level zero curve) to a given model. Distance transforms have bee successfully applied in the past to registration problems [53-55]. In a level set framework, Paragios has published several papers recently focusing on matching geometric shapes in a variational framework for global as well as local registration [56-58]. The first attempt at combining segmentation and registration in a single geometric deformable model framework might be attributed to Yezzi et al. in [59]. Their key observation is that multiple images may be segmented by evolving a single contour as well as the 
mappings of that contour into each image. In the context of level set framework, multiple recent works can be referenced that incorporate shape priors in the segmentation process as reviewed in [60]. The main trend of the reported efforts uses a shape model and incorporates a constraint in the energy of the geometric deformable model that forces the evolving contour to fit to the shape model $[56,61,62]$. In an effort to derive a rigorous and complete scheme, Paragios and Rousson [56] focused on the integration of a shape model, defined directly in a level set space, to derive a shape prior in an energetic form and integrate it with a data-driven variational segmentation framework. Applications of their combined registration and segmentation framework focused on the segmentation of physically corrupted of incomplete natural images.

In this chapter we selected to focus on recent works applied to segmentation and registration of medical images as this application typically involves tuning of a general framework to the specificity of the task at hand. We describe in details two different approaches in the next sections.

\section{B. Shape Priors into a Variational Segmentation Framework}

Several applications in medical imaging can benefit from the introduction of shape priors in the segmentation process using deformable models [49, 63-65]. Only few works on segmentation of medical imaging with level set framework attempted to perform simultaneous registration and segmentation into a single energy functional and we review three of them in this section.

We first review the work of Chen et al. $[60,66,67]$ that proposes a Mumford-Shah type energy functional plus a parameterized registration term embedded in a level set formulation for segmentation of brain MRI. Their approach consists of constraining the segmentation process with a level set framework by incorporating an explicit registration term between the detected 
shape and a prior shape model. They proposed two approaches either with a geodesic, gradientbased active contour or with a Mumford-Shah region-based functional.

The geodesic active contour minimizes the following functional:

$$
E(C, s, R, T)=\int_{0}^{1}\left\{g(|\nabla I|) C(p)+\frac{\lambda}{2} d^{2}(s R C(p)+T)\right\}\left|C^{\prime}(p)\right| d p
$$

with $C(p)$ a differentiable curve parameterized with $(p \in[0,1])$ defined on image $I, g$ a positive decreasing function, $(s, R, T)$ are rigid transformation parameters for scale, rotation and translation and $d(C(p))=d\left(C^{*}, C(p)\right)$ is the distance between a point $C(p)$ on the curve $C$ and the curve $C^{*}$ representing the shape prior for the segmentation task. A level set formulation is derived by embedding the curve $C$ into a level set function $\phi$ positive inside the curve. Let introduce the Heaviside function $H(z)=1$ if $z \geq 0, H(z)=0$ otherwise and the Dirac measure $\delta(z)=H^{\prime}(z)$ (with derivative in the distribution sense), the energy functional in Equation (31) is reformulated as:

$$
E(\phi, \mu, R, T)=\int_{\Omega} \delta(\phi)\left(g(|\nabla I|)+\frac{\lambda}{2} d^{2}(\mu R x+T)\right)|\nabla \phi|
$$

Four evolution equations are derived for the Euler-Lagrange system for temporal derivatives of the level set function $\phi$ and the rigid registration parameters $(\mu, R, T)$ with detailed numerical implementation described in [68]. In this paper the authors also report on experiments performed with this method to segment the endocardial borders of the left ventricle on an ultrasound image and segment the corpus callosum on mis-aligned functional MRI images in a time series.

In [60], the authors proposed a second functional for combining registration and segmentation in an implicit deformable model framework where the image gradient term is replaced by an homogeneity measure. Their approach is derived from the Mumford-Shah functional [36] in a 
similar fashion as described in the previous section of region-based level set methods. They proposed the following functional:

$$
\begin{aligned}
& E\left(\phi, c_{0}, C_{1}, \mu, R, T\right)=\alpha \int_{\Omega} H(\phi)\left(I-S^{+}\right)^{2}+\alpha(1-H(\phi))\left(I-S^{-}\right)^{2}+ \\
& \beta H(\phi)\left|\nabla S^{+}\right|+\beta(1-H(\phi))\left|\nabla S^{-}\right|+\delta(\phi) d^{2}(\mu R x+T)|\nabla \phi| d \Omega
\end{aligned}
$$

with $\left(S^{+}, S^{-}\right)$smooth approximations of the image $I$ on respectively $\{x / \phi(x)>0\}$ and $\{x / \phi(x)<0\}$ and $(\alpha, \beta)$ a set of positive parameters.

In a similar effort to combine registration and segmentation Paragios et al. [69] proposed a levelset approach for knowledge-based registration and segmentation of the left ventricle. In their method a level set framework was used to perform simultaneous segmentation of the epicardial and the endocardial surfaces of the myocardium muscle via coupling two level set functions $\left(\phi_{0}, \phi_{1}\right)$. The proposed functional is generalized as:

$$
E\left(\phi_{0}, \phi_{1}, A_{0}, A_{1}\right)=\alpha E_{G}\left(\phi_{0}, \phi_{1}\right)+\beta E_{R}\left(\phi_{0}, \phi_{1}\right)+\gamma E_{A}\left(\phi_{0}, \phi_{1}\right)+\delta E_{S}\left(\phi_{0}, \phi_{1}, A_{0}, A_{1}\right),
$$

This functional integrates four components:

(1) $E_{G}$ is a constraint on the regularity of the contour via minimization of its length.

(2) $E_{R}$ is an intensity-based region component. This component identifies the partition of the image into regions that maximizes the posterior segmentation probability given a priori gray level histogram distribution for the endocardium, the epicardium and the background;

(3) $E_{A}$ is an anatomy-driven constraint. It preserves the distance between the myocardium border surfaces within an admissible range of values.

(4) $E_{S}$ is a shape-driven global consistency constraint. This knowledge-based term performs a registration of the evolving contour to a prior shape model $\left(A_{0}, A_{1}\right)$ via rigid 
deformation. The prior shape models were defined in a pixel-wise stochastic level set representation [56].

The authors reported some experiments on segmentation of the endocardium from $2 \mathrm{D}$ cardiac MRI images. These experiments revealed that the anatomical constraint played a minor role in controlling the deformation of the segmenting surface and that the regularity term was overwritten by the shape prior term. Final segmentation results showed a reliable performance of the method but no quantitative validation was performed. It was pointed out by the authors that the integration of the different modules was difficult and that future refinements of the approach were considered such as the use of a single level set function for segmentation of the myocardium, and tracking contours in time by replacing the prior shape- model with the segmentation from the previous frame in the context of consecutive time frames segmentation over a cardiac cycle.

In two related papers, Paragios $[63,70]$ proposed modified versions of the method.

In [70] Paragios had proposed a version of the method where the regularity term consisted of a boundary component derived from gradient vector flow [71] to detect cardiac boundaries and curvature constraints on the segmented shape. No shape-driven constraint was proposed in this early work.

In a posterior work [63] Paragios modified the method for segmentation of the endocardial surface on ultrasound. The model was first modified to replace the regularity term $E_{R}$ by a boundary constraint $E_{B}$ derived from a geodesic active contour formulation [16]. The model was further modified to integrate temporal tracking of the segmented contours between consecutive time frames. A time-tracking constraint, in the form of a bounded error function using a robust norm $\rho$ was introduced as:

$$
\begin{aligned}
E_{T}\left(\phi^{t}, \phi^{t+1}, T\right)= & \int H\left(\phi^{t}\right) \rho\left(I^{t}-I^{t+1}(T)\right) d \Omega+ \\
& \int H\left(\phi^{t+1}\right) \rho\left(I^{t}\left(T^{-1}\right)-I^{t+1}\right) d \Omega
\end{aligned}
$$


where $H$ refers to the Heaviside function (equal to 1 for negative values and 0 on positive values) and $T$ is an optimal transformation to track the targeted structure of interest between to consecutive time-frames images $I^{t}$ and $I^{t+1}$ satisfying the visual consistency constraint:

$$
I^{t}(x, y) \approx I^{t+1}(x, y), \forall(x, y) / H\left(\phi^{t}(x, y)\right) \geq 0
$$

with $\phi$ defined with negative values inside the object to segment (i.e. the ventricle blood cavity in this case).

This work uses a shape-model defined in a level set framework. Several interesting recent efforts have focused on the use of level set framework for shape modeling and registration towards model-based shape-driven object extraction as reviewed in [58].

\section{Registering Contours for Multi-Modalities Segmentation}

In a recent paper Yezzi et al. [59] introduced a new variational deformable model framework that interleaves segmentation and feature-based registration for combined segmentation of a single organ from multiple screening modalities (e.g. skin surface from head CT and MRI).

They defined their problem as follows: They want to find closed surfaces $S$ and $\hat{S}$ to segment an object in images $I$ and $\hat{I}$ so that the curves, segmenting a same organ, are related through a geometrical mapping: $\hat{S}=g(S)$. The authors used rigid registration for the mapping (i.e. combination of rotation and translation) and defined the following coupled functionals for the surface $S$ and the registration parameters $g=\left[g_{1}, g_{2}, \ldots, g_{n}\right]$ :

$$
\begin{aligned}
& \frac{\partial S}{\partial t}=(f(x)+\hat{f}(g(x))) N-\kappa N \\
& \frac{d g_{i}}{d t}=\int_{S} \hat{f}(g(x))\left\langle\frac{\partial g(x)}{\partial g_{i}}, \hat{N}\right\rangle d A
\end{aligned}
$$


where $\kappa$ and $d A$ denote the mean curvature and area element of the surface $S,(N, \hat{N})$ are the unit normals of $(S, \hat{S})$. The registration vector is modelized as:

$$
g(x)=R x+T
$$

with $R=R_{X} R_{Y} R_{Z}$ is the combination of rotations around the three orthogonal axis $(X, Y, Z)$

defining the $3 \mathrm{D}$ domain, and $T=\left[T_{X}, T_{Y}, T_{Z}\right]$ is the translation vector in each axis direction.

The function $f(x)$ is defined in a homogeneity-based framework [40] as:

$$
f(x)=f_{\text {in }}(x)-f_{\text {out }}(x) \text {. }
$$

The functions $f_{\text {in }}$ and $f_{\text {out }}$ are defined as:

$$
\begin{aligned}
& f_{\text {in }}=(I-u)^{2} \\
& f_{\text {out }}=(I-v)^{2}
\end{aligned}
$$

where $u$ and $v$ denote the mean values of the image $I$ inside and outside the surface $S$. Analogous definitions of $\hat{f}$ with statistics on $\hat{I}$ are also derived.

The authors reported three experiments on simultaneous segmentation and registration of $\mathrm{MRI} / \mathrm{CT}$ images of the head and the spine both in $2 \mathrm{D}$ and 3D. Validation via visual inspection showed accurate contour extraction for these limited experiments.

\section{Review of Clinical Validations}

In this section we review in details several recent papers that apply level set segmentation and registration methods to medical images and provide a detailed validation of their method through a clinical study for qualitative and quantitative assessment of the accuracy of the method in assisting or performing a particular clinical diagnosis task. 


\section{A. Important clinical segmentation problems}

We introduce in some details the two major applications in the domain of segmentation of clinical images: Segmentation of the brain and segmentation of the left ventricular cardiac cavity.

\section{Segmentation of Brain Images}

The two major modalities used for brain screening are MRI and SPECT/PET.

Regarding MRI brain imaging, the paper in [72] gives a very nice review of the potential applications of MRI in quantification of brain disease. The brain cortex is a highly convoluted layer of gray matter that lies between the white matter and the cerebrospinal fluid (CSF). Clinical applications require reconstruction of the cerebral cortex from MRI data for:

(5) brain visualization

(6) analysis of brain geometry

(7) registration with other data sets (multimodality or repetitive scans of a single patient)

(8) surgical planning

(9) cortex mapping

The interface between WM and GM is clearly visible on T1-weighted MRIs. Difficulties of MRI segmentation arise from imaging noise, inhomogeneities, partial volume effects and the highly convoluted geometry of the cortex.

Regarding quantitative measurements of the brain anatomy [33] using MRI, whole brain volume, cortical gray matter volume, white matter volume, cortical surface area, cortical shape characteristics and cortical thickness map are among the most interesting to study brain anatomy and function. Such measurements can typically assist in characterizing, predicting or assessing neurological and psychiatric disorders via correlation to abnormality in the measurements. These measurements are all easily derived from the final level set function in a distance-preserving 
framework (typically ensured by reinitialization of the level set function during the iterative deformation process).

Two sources of MRI brain data for testing segmentation algorithms are available as open source databases on the web:

- The Internet Brain Segmentation Repository (IBSR) available at: http://www.cma.mgh.harvard.edu/ibsr/. This repository provides manually-guided expert segmentation results along with magnetic resonance brain image data. Its purpose is to encourage the evaluation and development of segmentation methods. The IBSR is supported by the National Institute of Neurological Disorders and Stroke at the NIH part of a grant that funds research in MR brain segmentation by researchers at Boston University, Draper Laboratory, Northeastern University, Massachusetts Institute of Technology, and Massachusetts General Hospital / Harvard Medical School. The IBSR is a World Wide Web resource providing access to magnetic resonance brain image data and segmentation results contributed and utilized by researchers from all over the world. Its purpose is to encourage the development and evaluation of segmentation methods by providing raw test and image data, human expert segmentation results, and methods for comparing segmentation results.

- The BrainWeb: Simulated Brain Database (SBD) from the McConnell Brain Imaging Centre Montréal Neurological Institute, at McGill University (http://www.bic.mni.mcgill.ca/brainweb/). This database contains a set of realistic MRI data volumes produced by an MRI simulator [73-76]. These data can be used by the neuro-imaging community to evaluate the performance of various image analysis methods in a setting where the truth is known. Currently, the SBD contains simulated brain MRI data based on two anatomical models: normal and multiple sclerosis (MS). Full 3-dimensional data volumes have been simulated using three sequences (T1-, T2-, 
and proton-density- (PD-) weighted) and a variety of slice thicknesses, noise levels, and levels of intensity non-uniformity. These data are available for viewing in three orthogonal views (transversal, sagittal, and coronal), and for downloading. Customization of the MRI simulations is also available allowing the user to run his own custom MRI simulation with any of several pulse sequences and source digital phantoms, and arbitrary values of the acquisition artifacts.

Regarding PET imaging, this modality uses small amounts of tracer drugs chemically attached to glucose or other compounds injected to the patient. As the tracer travels through the body, it emits signals and eventually collects in the organs targeted for examination. If an area in an organ is cancerous, the signals will be stronger than in the surrounding tissue. A scanner records these signals and transforms them into pictures of chemistry and function.

PET modality is used for brain screening for:

- diagnosis of Alzheimer's disease,

- location of tumors and scar tissue in the brain,

- location of seizures focus for patients with epilepsy,

- accurate assessment of tumor sites in the brain for surgery planning.

In practice, segmentation of PET brain data is difficult to perform in an automatic fashion because of the poor contrast and high noise level in the images. A standard procedure to delineate structures from PET brain images is to segment structures from the corresponding anatomical magnetic resonance images and then to superimpose them on the PET images. This method relies on an accurate registration between the two imaging modalities, handling the incongruity of structures and functions. Thresholding methods can provide a direct way to segment PET images with locally uniform radioactivity concentration and consistent structures. For more noisy data advanced methods such as deformable models have been 
studied for segmentation of cortical structure [77]. These methods must adapt to changes in individual radioactivity concentrations.

\section{Segmentation of Cardiac Images}

A reliable noninvasive imaging modality is essential for evaluating and monitoring patients with cardiac disease. Traditional screening techniques for quantitative assessment of cardiac function include the following modalities:

- Multi-Gated Angiography (MUGA): This is a slow screening modality that requires the injection of a radiopharmaceutical agent by a clinician. The purpose of MUGA screening is to examine the pumping function of the heart. After injection of a radioactive agent that labels red blood cells, a nuclear camera creates an image of the heart's chambers by counting these cells over numerous cardiac cycles. The information obtained can quantify ejection fraction but not ventricular volumes.

- Magnetic Resonance Imaging (MRI): Because of its complexity and even though MRI machines abound in the United States, cardiac MRI has largely been limited to university hospitals where there is a strong interest for research. This screening modality has proven very useful in evaluating patients' cardiac anatomy prior to surgery, in locating and characterizing cardiac tumors and in identifying and treating cardiac abnormalities for children with complex congenital heart disease. These clinical situations are relatively rare and cardiac MRI has yet to become a commonly used tool in clinical medicine.

- Computerized Tomography (CT): Multi-detector technology has made cardiac CT possible enabling angiography, perfusion and function studies. The main limitation of this screening modality remains the acquisition time with multi-row detectors, which may be solved with upcoming spiral CT, Electron Beam CT and Ultrafast CT technologies.

- Single Photon Emission Computed Tomography (SPECT): Commonly referred to as myocardial perfusion imaging, this technique is used to visualize myocardial blood flow 
distribution using intravenous injection of a radionuclide detected by single crystal gamma camera rotating around the patient's body. This modality can be used to assess ejection fraction and regional wall motion but cannot provide detailed views of anatomical structures.

- Positive Emission Tomography (PET): Similarly to SPECT, this technique visualizes myocardial blood flow using intravenous injection of positron-emitting tracers detected by multiple rings of stationary detectors encircling the patient's body to produce a series of multiple tomographic images encompassing the heart. Specific tracers have been developed for the evaluation and quantification of numerous physiological processes, including regional myocardial blood flow, metabolic processes, oxygen consumption, receptor activity, and membrane function. When comparing to SPECT, PET images have been shown to be more accurate in clinical studies but PET scanners remain costly and therefore less widely available than standard SPECT systems.

- Two-dimensional Echocardiography (2DE): Two-dimensional echocardiography is the fastest, least expensive, and least invasive screening modality for imaging the heart. Because of the three-dimensional structure and deformation of the heart muscle during the cardiac cycle, analysis of irregularly shaped cardiac chambers or description of valve morphology using 2D images is inherently limited. A second existing problem with 2DE, constrained to planar views, is that highly trained clinicians are required to perform the studies. But, despite its limited image quality and its limitation to planar acquisition, 2DE is one of the most popular cardiac screening modalities, available at hospitals, medical centers and cardiologist's offices. Critical information for assessment of cardiac pathology such as ventricular shape, wall deformation, valve motion, and blood flow (via Doppler acquisition mode) can be quickly assessed with this non-invasive, portable and relatively inexpensive screening modality. 
- Three-dimensional Echocardiography (3DUS): Three-dimensional ultrasound has been introduced in the late 1980s with off-line 3D medical ultrasound imaging systems. Many review articles have been published over the past decade, assessing the progresses and limitations of 3D ultrasound technology for clinical screening [78-81]. These articles reflect the diversity of 3D systems that were developed for both image acquisition and reconstruction. The evolution of $3 \mathrm{D}$ ultrasound acquisition systems can be divided into three generations:

- Freehand scanning. With freehand probes, planar images are acquired at arbitrary spatial positions and orientation with a $2 \mathrm{D}$ transducer. A positioning device, attached to the transducer, tracks its location in space and time during the acquisition. A three dimensional volume can then be reconstructed by associating each acquired image with its $3 \mathrm{D}$ spatial position and integrating in $3 \mathrm{D}$ space. This method offers a great scanning flexibility and provides high quality images. The principal limitations of the method are related to the precision of the positioning device (either mechanic, acoustic or magnetic) and the experience of the clinician in positioning the device to acquire sufficient amount of information for an accurate $3 \mathrm{D}$ reconstruction. These issues are most critical when scanning small moving structures such as valves and myocardium wall defect in cardiac applications.

- Mechanical scanning. With mechanical scanning, a 2D transducer is moved at regular intervals along a specified path, ensuring an accurate sampling of the volume to reconstruct with a probe whose position is controlled in space and time. The three most common scanning paradigms use linear, fan and rotational sweep. This technology provides a more accurate three-dimensional reconstruction of the anatomy than freehand scanning at the cost of a slower acquisition rate, and a smaller field of view. When screening the beating heart, gating is necessary for both modalities in order to acquire data corresponding to similar instants in the cardiac 
cycle, so that the anatomy is approximately the same, assuming a periodic movement, for each planar view.

- Phased arrays. Real-time three-dimensional (RT3D) volumetric imaging is the only true three-dimensional ultrasound modality [82-87]. This technology, pioneered by Dr. Olaf Von Ramm and Dr. Stephen Smith at Duke University, is fundamentally different from the former generations of $3 \mathrm{D}$ systems as a volume is acquired with a 2D array of pulse transmitter and receiver elements, enabling the cardiologist to view moving cardiac structures from any given plane in real-time [82, 87-91]. Electronic scanning controls the acquisition depth allowing real-time signal acquisition through a pyramidal shape volume. This existing technique is still limited by hardware component size and speed. The low spatial resolution and high noise level have prevented this technology from meeting its initial expectation and reaching its full potential. It remains, nevertheless, the only true $3 \mathrm{D}$ ultrasound modality that can enable accurate temporal visualization of cardiac deformation during a single cardiac cycle.

In current clinical practice, cardiologists use anatomical images from CT, US and MRI modalities to quantify cardiac function through measurements of ventricular volumes at end diastole (ED) which corresponds to the end of the blood filling phase in the cardiac cycle, and end systole (ES) which corresponds to the end of the blood ejection phase, stroke volume (SV) which is equal to the difference of blood volumes and ED and ES and ejection fraction (EF) which is equal to SV over ED volume. These measurements are performed via simple visual inspection or manual tracing of 2D slices extracted from the $3 \mathrm{D}$ data. A second phase of analysis of the images aims at analyzing myocardium wall deformation and localization of abnormalities. Physiological images provided by MUGA, SPECT and PET/SPECT modalities aim at quantifying myocardium tissue blood perfusion for localization of ischemic tissue. 
All these diagnostic tasks require the intervention of an expert cardiologist familiar with the modality for quick visual inspection. A more detailed processing of the data always require segmentation of the myocardium tissue versus the blood pool and sometimes the outside tissues. Few automated segmentation algorithms, with minimal manual intervention, are available on clinical consoles to assist the segmentation task with a significant saving of time. Segmentation of cardiac images is still a very active research area and level set segmentation methods have proved in the recent years to offer a very flexible three-dimensional tool that can handle the volumetric and dynamic nature of the data.

\section{B. Free Software for Level set Segmentation}

\section{Snake Automatic Partitioning (SNAP)}

This software was developed by the Medical Image Display and Analysis Group at the University of North Carolina and is available for download at midag.cs.unc.edu. SNAP is a segmentation tool for volumetric image data using 3D level set methods with either a region-probability deformable model or a gradient-based deformable model framework. Some interaction with parameter settings of the segmentation method and prior-filtering is available. Interactive visualization of the deformation process in provided.

\section{Insight Segmentation and Registration Toolkit (ITK)}

The National Library of Medicine Insight Segmentation and Registration Toolkit (ITK) is an open-source software system to support the Visible Human Project. The toolkit is available for free download at www.itk.org. Under active development, ITK employs leading-edge segmentation and registration algorithms in multiple dimensions. The Insight Toolkit was

developed by six principal organizations, three commercial (Kitware, GE Corporate R\&D, and Insightful) and three academic (UNC Chapel Hill, University of Utah, and University of 
Pennsylvania). Additional team members include Harvard Brigham \& Women's Hospital, University of Pittsburgh, and Columbia University. The funding for the project is from the National Library of Medicine at the National Institutes of Health. NLM in turn was supported by member institutions of NIH (see sponsors). Several level set segmentation methods are implemented in this toolkit including: fast marching methods, shape detection segmentation, geodesic active contours, threshold level set, canny-edge level set and Laplacian level set methods.

\section{Review of Applications to Clinical Studies}

\section{Robust Adaptive Segmentation of 3D Medical Images with Level Sets}

This work was published by Baillard, Barillot and Bouthemy in [30].

Method: The proposed method uses a 3D level set algorithm with the introduction of an adaptive adjustment of the time step and the external propagation force at each iteration. A region-based force is derived from intensity probability density functions over the data. Assumptions are made on the input data which is modeled as a mixture of distributions. Mixture of Gaussian distributions for MRI and Gaussian and Rayleigh distributions for ultrasound data are validated through two experiments. Each distribution defines a class $C_{k}$ through a parameter vector that contains the distribution parameters and the probability $p_{k}$ that a voxel belongs to class $C_{k}$. The parameters vector is estimated from the data using the stochastic expectation-maximization (SEM) algorithm [92], which is a stochastic version of the EM algorithm that utilizes

probabilistic learning stage. Advantages of the SEM over the EM algorithm include: (1) Only an over estimation of the number of classes is required, (2) it is less dependent on the initialization. 
The stopping criterion for the deformation process is based on the stabilization of the average segmented volume size.

Experiments: Experiments were performed on brain MRI volumes. The statistical model was initialized with 7 classes.

1. A first experiment used simulated brain MRIs from the MNI group [93]. Brain MRI volumes of size $(181 \times 217 \times 181)$ simulating WM, GM and CSF were generated under noiseless conditions and three different combinations of noise and inhomogeneities. The segmentation method was applied to extract together GM and WM volumes. Initialization was performed by defining a large cube of size $(100 \times 70 \times 70)$ inside the data volume. Gaussian distribution parameters for WM + GM were automatically estimated prior to segmentation. Quantitative validation was performed using overlapping measurements [94] between the result and the known ground truth on these phantom data sets. The measures included estimation of the number of true-positive (TP) truenegative (TN), false-positive (FP) and false negative (FN) voxels and the definition of the following measures:

$$
\begin{array}{ll}
\text { sensitivity } & =T P /(T P+F N) \\
\text { specificity } & =T N /(F P+T N) \\
\text { total performance } & =(T P+T N) /(T P+F P+T N+F N)
\end{array}
$$

These measures are very helpful to assess the global performance of a segmentation method such as under-segmentation characterized by a low sensitivity of over-segmentation characterized by a low specificity. The total performance of the proposed algorithm stabilized around $98.3 \%$ under all noise conditions. The authors further compared their segmentation performance to morphological operators performance and reported an improvement of sensitivity performance with the level set method.

2. A Second set of experiments with a database of 18 real brain MRIs of size $(256 \times 256 \times 176)$ was performed. Results reported a $94 \%$ success ratio of segmentation convergence (one case failed), requiring on average 1,000 iterations. Segmentation of individual tissue classes (WM, 
GM and CSF) required a coarse approximation of tissue segmentation for class definition and computation of a priori statistical models.

Limitations: The proposed segmentation method has a performance limited by the fact that the SEM algorithm does not guarantee an optimal solution. In practice, an initial partitioning roughly representative of the inside and outside distributions of the organs to segment lead to a correct solution. This means that tissue classes need to be initialized with relatively accurate average intensity values.

\section{Topology Preserving Geometric Deformable Models for}

\section{Brain Reconstruction.}

This research work was published by Han, Xu and Prince in [72].

Method: The authors proposed a 3D level set segmentation method with a speed term based on binary flow forces, mean curvature flow and gradient vector flow. The originality of the method was to focus on the topology of the evolving front and use the notion of simple points and update the front deformation only at their locations. Given a set of points defining a 3D surface, a point is simple if its addition or removal from the object does not change the topology of either the object or the background. The topology of an object is defined through its number of connected components, cavities and handles. The algorithm was implemented with a narrow-band update and 3D level set fast marching propagation scheme for computational efficiency. The final object surface, which corresponds to the zero-level of the level set function is extracted with a connectivity consistent marching cubes algorithm (CCMC) [95, 96]. This algorithm is a modification of the standard marching cubes algorithm where the resolution of ambiguous cubes depends on pre-defined digital connectivity rules.

Experiments: The authors performed two sets of experiments on brain MRIs.

1. A first experiment compared visual quality of segmented data with the topology preserving deformable model to a standard geometric deformable model and a parametric deformable model 
using the same initialization scheme. Results showed very similar looking surfaces for the three methods but close inspection revealed critical differences:

- The parametric deformable model surface had self-intersection points,

- The number of handles with the simple geometric deformable model was 40 versus 0 for the two other methods (corresponding to the correct manifold).

In this experiment the authors also provided an example from a brain MRI data set where part of the WM seemed to display a handle when viewed in 3D corresponding to an incorrect topology. This type of errors, mostly due to MRI noise, can only be corrected with a topology preserving segmentation method such as the proposed level set framework.

2. A second set of experiments employed 21 T1-weighted MRI volumes with voxel size $(0.9375 \times 0.9375 \times 1.5 \mathrm{~mm})$ from the public database of the Baltimore study on aging [97]. Volumes were pre-processed to remove extra-cranial tissues, cerebellum and brain stem. The experiments were performed with digital connectivity defined as: 18-connectivity for the object (WM) and 6-connectivity for the background. The experiments focused on the extraction of central cortical surfaces. Prior to segmentation, the volumes were processed with fuzzy connectedness [98] for labeling into memberships to different tissue types. The result of this labeling was used as an initial segmentation of the WM and used to fill the ventricles and the sub cortical structures (including the thalamus, hypothalamus, caudate nucleus and putamen). The filled WM volume was then binarized via thresholding of the fuzzy values at 0.5 . This binary volume was further processed for topology correction with a multiscale graph-based algorithm [99]. The CCMC was then used to extract the WM surface of the volume. At this point, the WM surface was used as the initial level zero of the level set segmentation to extract three cortical surfaces: WM/GM surface, central cortical surface and pial (CM/CSF) surface.

Segmentation accuracy was assessed through error measurements at 10 landmark points manually selected on major sulci and gyri on six MRI cases. Landmark error was measured as the minimum distance between the landmark points and the segmented surfaces. The overall average 
error was $0.87 \mathrm{~mm}$ (std $0.5 \mathrm{~mm}$ ) outperforming a previous method from the same group based on a parametric deformable model that produced an average error of $1.22 \mathrm{~mm}$ (std $1.01 \mathrm{~mm})$ [100]. Visual inspection of the segmented data did not reveal any self-intersection on the extracted surfaces. The algorithm computational time was about 40 minutes on a SGI O2 workstation for reconstruction of the three surfaces. This performance compares favorably to typical deformable model algorithms with arbitrary initialization as claimed by the authors.

Limitations: The main limitation of this algorithm is the involvement of the pre-processing for initialization of the WM that make the process difficult to reproduce.

\section{Segmentation and Measurement of the cortex from 3-D MR images using coupled-surfaces propagation}

This research was published by Zeng, Staib, Schultz and Duncan in [33].

Method: The authors proposed the segmentation and measurement of the cortical GM thickness from brain MRI data with a level set method using coupled-surfaces propagation. As stated by the authors, coupling surfaces can prevent two problems:

- The inner cortical surface can collapse with the CSF due to higher contrast at the $\mathrm{CSF} / \mathrm{GM}$ interface than at the WM/GM interface.

- The presence of eye sockets with no CSF signal can drive the outer cortical surface to expend outward from the brain.

Constraining the cortical thickness during the segmentation process prevent the collapse of leakage of the surfaces.

The level set segmentation method with surface coupling is described in the first Section of this chapter. The traditional gradient features in the speed term were replaced with tissue interface probability measurements based on statistical priors summarized here. The statistical models are based on the assumption of Gaussian independent distribution functions of voxel intensities in MRI volume data for WM, GM and CSF. Let assume the presence of two tissue types A and B in 
the data with independent Gaussian probabilities $G\left(\mu_{A}, \sigma_{A}\right)$ and $G\left(\mu_{B}, \sigma_{B}\right)$. For each voxel s, a set of 26 immediate 3D-neighborhood voxels can be defined. For each neighbor voxel, a normal direction $\theta$ along the line passing through the center voxel and the neighbor voxel is computed which defines a plane that separates the neighborhood into two regions $\left(R_{1}, R_{2}\right)$. The probability of the center voxel belonging to an interface between the two tissue types $(A, B)$ is then computed as:

$$
p_{A B}(\theta)=\prod_{r \in R_{1}} \frac{1}{\sqrt{2 \pi} \sigma_{A}} \exp \left(\frac{-\left(I(r)-\mu_{A}\right)^{2}}{\sigma_{A}{ }^{2}}\right) \prod_{r \in R_{2}} \frac{1}{\sqrt{2 \pi} \sigma_{B}} \exp \left(\frac{-\left(I(r)-\mu_{B}\right)^{2}}{\sigma_{B}{ }^{2}}\right)
$$

where $I(r)$ is the intensity value of the data at neighbor voxel $r$. The final density probability at voxel $s$ is set to the highest value of $p_{A B}(\theta)$ over all the 26 directions. An illustration of a feature map based on this tissue interface probability measure is provided on a single brain MRI slice computed with $\left(R_{1}, R_{2}\right)$ containing only one voxel. The example illustrates well the better performance of the interface probability feature at extracting locations of tissue transitions for WM, GM and CSF when compared to standard gradient maps.

Experiments: Validation was performed on T1-weighted MRIs. The segmentation process was initialized with several pairs of concentric spheres with a constraint on starting inside the WM for robust behavior.

1. A first experiment used simulated MRI data from the McConnell Brain Imaging Center at the Montreal Neurological Institute [93]. The authors simulated T1-weighted brain MRIs with 3\% noise and $1 \mathrm{~mm}^{3}$ voxel size. Distance range between two surfaces was set to [1.5mm $5.5 \mathrm{~mm}$ ] leading to bandwidth ranges for the inner and outer surfaces of [-3mm $6 \mathrm{~mm}]$ and $[-6 \mathrm{~mm} 3 \mathrm{~mm}]$ respectively. Segmentation was validated by comparing the binary segmented volumes to the thresholded membership values of the corresponding voxels (with reference to WM, GM and 
CSF) above 0.5 from the data base ground truth data. The authors computed TP, FP rates and total volume ratios (VR) to compare segmented volumes and thresholded ground truth volumes. Results with [TP FP VR] were: [92.3\% 2.0\% 96.3\%] for (WM+GM), [92.8\% 6.0\% 103.2\%] for cortical GM and [92.4\% 3.3\% 98.1\%] for WM showing good performance of the algorithm in isolating brain tissue and segmenting the cortex.

2. A second experiment was performed using $20 \mathrm{~T} 1$-weighted spoiled gradient MRIs of normal brain subject from the Internet Brain Segmentation Repository (IBSR) of the Center for Morphometric Analysis at the Massachusetts General Hospital [101]. Cases were acquired with two different scanners and all registered to a standard 3D brain coordinate system. Manual segmentation from medical experts wais available. An overlap metric was defined by the IBSR to evaluate the performance of automatic segmentation methods, measuring the ratio $\mathrm{TP} /(1+\mathrm{FP})$. Such ratio ranges from 0 for no agreement to 1 for perfect agreement with the manual segmentation considered as the ground truth. MRI data was interpolated from $3 \mathrm{~mm}$ thick coronal slices (as provided) to $1 \mathrm{~mm}$ thickness, achieving isotropic voxels. The GM overlap metric on the whole brain was 0.657 . It outperformed other reported segmentation performances on the same data sets from [102] with overlap ranking from 0.47 to 0.56 . Overlap metric specifically computed on the cerebral cortex (excluding brain stem and cerebellum) was further improved to 0.701. The authors pointed out that if applied to the phantom data, the overlap metric was 0.875 that compared to the manual segmentation variability of 0.876 reported in the IBSR database.

3. A third experiment was performed for a study of the frontal lobe anatomy on 7 highresolution MRI data sets acquired with SPGR with isotropic voxel size of $1.2 \mathrm{~mm}^{3}$. The patient population of this study included young autistic and control subjects for comparison of frontal lobe volumes. The MRI volumes were pre-processed for inhomogeneity correction. Segmentation was performed with coupled level set functions and the frontal lobe was manually isolated with anatomical landmarks. Segmentation accuracy was compared to expert manual tracing. TP and FP on entire frontal lobe averaged $94.1 \%$ and $2.1 \%$. TP and FP for cortical GM on frontal lobe 
averaged $86.7 \%$ and $20.8 \%$. The authors further evaluated the reliability statistics on the volume measurements obtained on the segmented frontal lobe volumes using the method proposed by Schultz and Chakraborty [103]. The agreement between the expert tracing and the level set segmentation method was very strong for both the entire frontal lobe and only the GM but the level set algorithm systematically under estimated the frontal lobe volume with a mean difference of $4 \%$.

4. In a fourth experiment, regional cortical thickness was quantitatively analyzed on 7 highresolution MRI data sets acquired with SPGR and isotropic voxel size of $1.2 \mathrm{~mm}^{3}$. Cortical measurements were performed in 4 lobes to compare level set segmentation and expert manual tracing. The authors first compared the mean thickness of each lobe to the results from the study of 63 post-mortem males by Pakkenberg and Gundersen [104]. The new segmentation produced similar results with a frontal cortex thicker than the occipital cortex. On overall, post-mortem data was $5-14 \%$ thinner than the new results while variability of thickness was equal to $1.5 \mathrm{~mm}$ for both studies. Statistical tests also showed that frontal and temporal lobes were thicker than parietal and occipital lobes.

This method relies on prior statistics for WM, GM and CSF for the construction of interface probability maps. The method also requires manual initialization by clicking center points of concentric spheres on slices. The authors demonstrated on one example that the number of spheres or their localization does not have a critical influence on the accuracy or reproducibility of the method. On the other hand major advantages of the method include the fact that it does not require stripping of non-brain data, its performance was demonstrated on a wide range of MRI image quality and it offers fast computation times compared to existing methods.

\section{Segmentation Of RT3D Ultrasound With Geodesic Deformable Models}

This research was published by Corsi, Saracino, Sarti and Lamberti in [105]. 
Method: The proposed method uses a geodesic deformable model as defined in Equation (17), with weighting parameters associated with the two terms and a function $g($ defined as in [8]. To handle the noisy nature of the ultrasound data and the poor definition of the myocardium wall borders in some frames, the authors did not use any inflationary force in the speed term. This type of model requires an initialization close to the final endocardial surface to ensure that the moving front is attracted to local high data gradient locations. The algorithm was implemented with the narrow-band technique for computation efficiency.

Experiments: The authors performed 3D segmentation of echocardiographic real-time three dimensional (RT3D) ultrasound data for extraction of ventricular volumes. Prior to segmentation, a clinician roughly defined endocardial contours on a limited set of short-axis views. The polygonal surface defined by this manual tracing was then used to initialize the level set segmentation process. Deformation of the moving front required about 40 s for a single volume on a PC and initial manual tracing less than a 1 minute. The authors performed three experiments for assessment of the method accuracy and robustness to the initialization:

1. In a first experiment the authors performed multiple segmentation of the same RT3D volume using manual initialization from six different users. Comparing volume measurements to precise manual tracing they report a mean square error of $3.8 \%$ and a maximum error of $4.38 \%$. They concluded from these results that the segmentation is rather sensitive to the manual initialization.

2. In a second experiment, the authors segmented in-vitro phantom data of eighteen balloons filled with water and immerged in a tank of water. To take into account the sensitivity of the segmentation technique to the initialization, two operators performed two separate tracings and the segmentation was run with these different initializations. A linear regression coefficient of 0.99 was reported between true volume values and measurements from seventy level set segmentations. The standard error of estimate was equal to $9.35 \mathrm{ml}$, the average error of measurement was $-2.63 \mathrm{ml}$ (std $10.81 \mathrm{ml}$ ). Intra-observer variability was estimated for each 
operator as: $1.66 \%$ and $-1.36 \%$. Inter-observer variability was estimated as $1.63 \%$. This experiment reported a maximum error of measurement of $40 \mathrm{ml}$ for large volumes (above $200 \mathrm{ml}$ ). Errors of measurements decreased significantly with balloons true volumes suggesting that ventricular volumes can be accurately measured with this technique for physiological volume ranges.

3. An in-vivo study was also performed on about 18 RT3D cases (exact number not specified in the paper) using again two different manual tracings from two operators. The ventricular volume range for this study was $[151 \mathrm{ml}-467 \mathrm{ml}]$ which suggest that it included dilated cardiomyopathy patients with enlarged ventricular cavity. Volume measurements were compared to measurement from manual tracing on MRI. Linear regression was performed with a correlation coefficient of 0.97. The standard error of estimate was $20.13 \mathrm{ml}$, average error was $-15.58 \mathrm{ml}$ (std $20.55 \mathrm{ml}$ ). Intra-observer variability was $0.16 \%$ and $-2.04 \%$, and inter-observer variability was $-2.16 \%$. Ejection fraction measurements were performed on nine RT3D cases. The correlation coefficient was 0.87 when comparing to MRI measurement. The main limitation of this study is the absence of testing on normal physiological ventricular volume in the range $[40 \mathrm{ml} 150 \mathrm{ml}]$ for which the behavior of the level set segmentation can be significantly different as these volumes are much smaller.

\section{Segmentation Of RT3D Ultrasound With Implicit Deformable Models Without Gradients}

This research was published by Angelini, Holmes, Laine and Homma in [43].

Method: This study focused on the same clinical problem as the previous study for segmentation of echocardiographic RT3D ultrasound data. The proposed method uses the homogeneity-based implicit deformable model proposed by Chan and Vese in [40] as an extension of the MumfordShah segmentation functional. Motivations for selection this method include robustness with arbitrary initialization of the object anywhere in the image, topology adaptation for multi-object 
segmentation (for potential co-segmentation of ventricles and atria for example), self-adaptation of the deformation flow to inward and outward flows. Minor modifications of the method were performed to adapt the design to the specificity of the $3 \mathrm{D}$ ultrasound data. The homogeneity terms from Equation (28) were weighted by the mean intensity value as:

$$
E(C)=\int_{\text {insideC }}\left(\frac{I-c_{0}}{c_{0}}\right)^{2} d \Omega+\int_{\text {outsidec }}\left(\frac{I-c_{1}}{c_{1}}\right)^{2} d \Omega .
$$

A similar approach was followed by Lin et al. [44] for segmentation of 3D echocardiographic data where they normalized the homogeneity term by the variance of the data inside and outside the object segmented, after pre-processing with multi-scale Gaussian filtering.

No and the weight associated with the definition on the segmentation process. Parameters were set to $v=0$ (no constant inflation force was used), $\mu=1, \lambda_{1}=0.25, \lambda_{2}=0$ (no homogeneity constraint on the outside of the ventricle to reduce the effect of the noisy myocardium texture), $\Delta \mathrm{x}=\Delta \mathrm{y}=\Delta \mathrm{z}=1, \Delta \mathrm{t}=\min (\Delta \mathrm{x}, \Delta \mathrm{y}, \Delta \mathrm{z}) /\left|\mathrm{v}+\mu+\lambda_{1}+\lambda_{2}\right|$ (to respect CFL condition with explicit numerical scheme). The system was let to deform over 20 iterations.

Experiments: A clinical study was performed on 10 patients with primary hypertension to segment both right and left ventricular volumes. A 2D parametric deformable model and a 3D Level set deformable model were used for segmentation of the ultrasound data after denoising with a spatio-temporal brushlet expansion [106]. The model was initialized with a cone which dimensions were defined manually on slices at the base and apex. Manual tracing on ultrasound data was performed by an expert clinician. MRI data were also acquired on the patients and manually traced by a second expert. Absolute errors of measures were computed for RV and LV ejection-fraction. Mean-error values and standard deviation over the ten cases for the two ventricles were equal to [Mean Std Min Max]: [8.6\% 5.7\% 17.8\% 0.3\%] for Manual tracing on ultrasound vs. MRI, $\left[\begin{array}{lllll}4.9 \% & 4.1 \% & 12.21 \% & 0.2 \%\end{array}\right]$ for $2 \mathrm{D}$ parametric deformable model vs. MRI, $\left[\begin{array}{llll}4.6 \% & 4.2 \% & 13.9 \% & 0.8 \%\end{array}\right]$ for $3 \mathrm{D}$ level set deformable model vs. MRI. Improvement of 
correlation measurements with deformable models (with good statistical significance) was reported when compared to MRI as well as better accuracy with a Bland-Altman analysis. The study concluded that errors of EF measurements using deformable models were within the range of inter- and intra- observer variability for both ventricles and compared favorably to similar studies performed by other groups using RT3D ultrasound for quantification of cardiac function. Manual tracing measures were significantly less reliable with large standard deviation of errors and low correlation coefficients. Finally, the 3D level set deformable model achieved the highest degree of accuracy, which can be explained by a more accurate segmentation of small and distorted ventricular shapes when integrating the third spatial dimension.

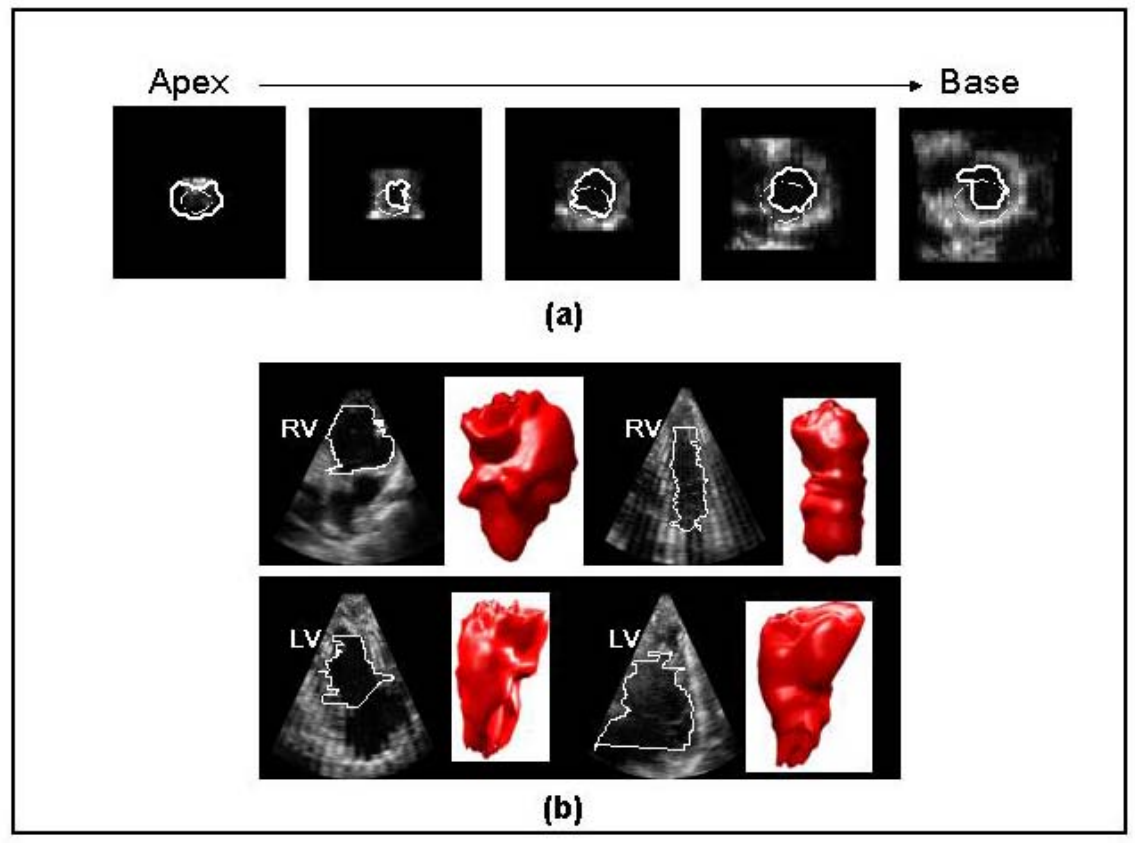

Figure 6: Segmentation of right and left ventricular volumes with a 3D implicit deformable model on RT3D ultrasound data. (a) Initialization of the segmentation with a cone shape surface (dashed line) and final position of the contour (continuous line) on the endocardial surface. (b) Illustration of diversity of right and left ventricular shapes and sizes extracted for the clinical study reported in [43]. 


\section{Conclusion}

Level set methods for segmentation and registration of medical images have been the focus of intense research for the past decade producing very promising results. Major advantages of the method include its robustness to noisy conditions, its aptitude in extracting curved objects with complex topology and its clean numerical framework of multi-dimensional implementation. Despite their success, these methods still need to be refined to address two limitations:

(1) computation time needs to be further reduced, for viability of the method in clinical application where interactivity (and therefore close to real time computation) is critical. This optimization will have to handle the constant increase in data size observed in medical imaging applications with improvements of spatial resolution, temporal resolution and now the introduction of combo scanners such as PET/CT machines.

(2) Robustness to variation in image quality and organ anatomy needs to be studied. Unfortunately, the methods described in this chapter were only rarely validated in clinical studies. On the other hand it is well known that these methods require tuning of their parameters to adapt to the nature of the image data to segment. In that optic, it is therefore critical to evaluate robustness of the performance on a set of data that covers the range of quality encountered in clinical practice for a particular examination. For methods based on shape models, it is also critical to test the method on a variety of abnormal (e.g. disease) cases that differ from the average anatomy that they typically represent. Such validation for medical application should always clearly specify the context of the problem at hand in terms of anatomy of interest (e.g. endocardial surface of myocardium muscle), imaging modality (e.g. three-dimensional real-time ultrasound) and clinical application targeted (e.g. quantification of volume). Only in this context can a segmentation method be really tuned, tested and validated for clinical application [107]. 


\section{E. References}

[1] T. McInerney and D. Terzopoulos, "Deformable models in medical image analysis: A survey," Medical Image Analysis, vol. 1, pp. 91-108, 1996.

[2] M. Kass, A. Witkin, and D. Terzopoulos, "Snakes: Active contour models," International Journal of Computer Vision, vol. 1, pp. 321-331, 1987.

[3] D. N. Metaxas, Physics-based deformable models. Applications to computer vision, graphics and medical imaging, 1997.

[4] C. Xu, D. Pham, and J. Prince, "Image segmentation using deformable models.," in Handbook of Medical Imaging, vol. 2: SPIE, 2000, pp. 129-174.

[5] T. McInerney and D. Terzopoulos, "Topologically adaptable snakes," in the Proceedings of 5th International Conference on Computer Vision, pp. 840-845, 1995.

[6] R. Durikovic, K. Kaneda, and H. Yamashita, "Dynamic contour: a texture approach and contour operations," The Visual Computer, vol. 11, pp. 277-289, 1995.

[7] S. Osher and J. A. Sethian, "Fronts propagating with curvature-dependent speed: Algorithms based on Hamilton-Jacobi formulations," Journal of Computational Physics, vol. 79, pp. 12-49, 1988.

[8] R. Malladi, J. A. Sethian, and B. C. Vemuri, "Shape modeling with front propagation: A level set approach," IEEE Transactions on Pattern Analysis and Machine Intelligence, vol. 17, pp. 158-175, 1995.

[9] J. S. Suri, K. Liu, S. Singh, S. N. Laxminarayan, X. Zeng, and L. Reden, "Shape recovery algorithms using level sets in 2-D/3-D medical imagery: a state-of-theart review," IEEE Transactions on Information Technology in Biomedicine, vol. 6, pp. $8-28,2002$.

[10] J. S. Suri and K. Liu, "Level set regularizers for shape recovery in medical images," in the Proceedings of IEEE Symposium on Computer-Based Medical Systems, pp. 369 -374, Bethesda, MD, USA, 2001.

[11] J. Montagnat, H. Delingette, and N. Ayache, "A review of deformable surfaces: topology, geometry and deformation," Image and Vision Computing, vol. 19, pp. 1023-1040, 2001.

[12] V. Caselles, F. Catte, T. Coll, and F. Dibos, "A geometric model for active contours," Numerische Mathematik, vol. 66, pp. 1-31, 1993.

[13] J. Sethian, "Numerical algorithms for propagating interfaces: Hamilton-Jacobi equation and conservation laws," Journal of Differential Geometry, vol. 31, pp. 131-161, 1990.

[14] J. A. Sethian, Level Set Methods and Fast Marching Methods: Evolving interfaces in computational geometry, fluid mechanics, computer vision, and materials science. Cambridge, UK: Cambridge University Press, 1999. 
[15] S. Kichenassamy, A. Kumar, P. Olver, A. Tannenbaum, and A. Yezzi, "Gradient flows and geometric active contour models," in the Proceedings of IEEE International Conference in Computer Vision, pp. 810-815, Cambridge, MA, USA, 1995.

[16] V. Caselles, R. Kimmel, and G. Sapiro, "Geodesic active contours," in the Proceedings of International Conference on Computer Vision, pp. 694-699, Cambridge, MA, USA, 1995.

[17] A. Yezzi-Jr, S. Kichenassamy, A. Kumar, P. Olver, and A. Tannenbaum, "A geometric snake model for segmentation of medical imagery," IEEE Transactions on Medical Imaging, vol. 16, pp. 199-209, 1997.

[18] R. Malladi, R. Kimmel, D. Adalsteinsson, G. Sapiro, V. Caselles, and J. A. Sethian, "A geometric approach to segmentation and analysis of 3D medical images," in the Proceedings of Workshop on Mathematical Methods in Biomedical Image Analysis, pp. 244-252, 1996.

[19] W. J. Niessen, B. M. t. H. Romeny, and M. A. Viergever, "Geodesic deformable models for medical image analysis," IEEE Transactions on Medical Imaging, vol. 17, pp. 634 - 641, 1998.

[20] K. Siddiqi, Y. B. Lauziere, A. Tannenbaum, and S. W. Zucker, "Area and Length Minimizing Flows for Shape Segmentation," IEEE Transactions on Image Processing, vol. 7, pp. 433-443, 1998.

[21] T. Deschamps, "Curve and Shape Extraction with Minimal Path and Level-Sets techniques. Applications to 3D Medical Imaging," in Mathématiques Appliquées et Traitements d'Images. Paris, France: Université de Paris Dauphine, 2001.

[22] A. Yezzi-Jr., A. Tsai, and A. Willsky, "A statistical approach to snakes for bimodal and trimodal imagery," in the Proceedings of IEEE International Conference on Computer Vision, pp. 898-903, Kerkyra, Greece, 1999.

[23] N. Paragios and R. Deriche, "Geodesic active contours and level sets for the detection and tracking of moving objects," IEEE Transactions on Pattern Analysis and Machine Intelligence, vol. 22, pp. 266-280, 2000.

[24] A. Sarti and R. Malladi, "A Geometric Level Set Model for Ultrasounds Analysis," University of California, Berkeley LBNL-44442, 1999.

[25] Y. Jin, A. Laine, and C. Imielinska, "An adaptive speed term based on homogeneity for level-set segmentation," in the Proceedings of SPIE Medical Imaging Conference, pp. 383-390, San Diego CA, USA, 2002.

[26] P. K. Saha, J. K. Udupa, and D. Odhner, "Scale-based fuzzy connected image segmentation: theory, algorithms, and validation," Computer Vision and Image Understanding, vol. 77, pp. 145-174, 2000.

[27] A. Sarti, R. Malladi, and J. A. Sethian, "Subjective surfaces: a geometric model for boundary completion," International Journal of Computer Vision, vol. 46, pp. 201-221, 2002.

[28] J. S. Suri, "Leaking prevention in fast level sets using fuzzy models: an application in MR brain," in the Proceedings of Information Technology Applications in Biomedicine, pp. 220-225, Arlington, VA, USA, 2000.

[29] J. S. Suri, "Leaking prevention in fast level sets using fuzzy models: an application in MR brain," in the Proceedings of IEEE EMBS International 
Conference on Information Technology Applications in Biomedicine, pp. 220 225, Arlington, VA, USA, 2000.

[30] C. Baillard, C. Barillot, and P. Bouthemy, "Robust Adaptive Segmentation of 3D Medical Images with Level Sets," INRIA, Rennes, France, Research Report Nov. 2000.

[31] M. E. Leventon, W. E. L. Grimson, and O. Faugeras, "Statistical shape influence in geodesic active contours," in the Proceedings of IEEE Conference on Computer Vision and Pattern Recognition, pp. 316-325, 2000.

[32] M. Leventon, E. Grimson, O. Faugeras, S. Wells, and R. Kikinis, "Knowledgebased segmentation of medical images," in Geometric Level Set Methods in Imaging, Vision and Graphics, N. P. S. Osher, Ed. New York: Springer Verlag, 2003.

[33] X. Zeng, L. H. Staib, R. T. Schultz, and J. S. Duncan, "Segmentation and measurement of the cortex from 3-D MR images using coupled-surfaces propagation," IEEE Transactions on Medical Imaging, vol. 18, pp. 927-937, 1999.

[34] J. Gomes and O. Faugeras, "Segmentation of the inner and outer surfaces of the human cortex: an approach based on partial differential equations," in the Proceedings of 22nd Annual International Conference of the IEEE Engineering in Medicine and Biology Society, pp. 1764-1774, Chicago, IL, USA, 2000.

[35] G. Barles, H. M. Soner, and P. E. Souganidis, "Front propagation and phase field theory," SIAM Journal of Control and Optimization, vol. 31, pp. 439-469, 1993.

[36] D. Mumford and J. Shah, "Boundary detection by minimizing functional," in the Proceedings of International Conference on Computer Vision and Pattern Recognition, pp. 22-26, San Francisco, CA, USA, 1985.

[37] T. F. Chan and L. A. Vese, "Active contour and segmentation models using geometric PDE's for medical imaging," in Geometric Methods in Bio-Medical Image Processing, Mathematics and Visualization, R. Malladi, Ed.: Springer, 2002.

[38] T. F. Chan and L. A. Vese, "A level set algorithm for minimizing the MumfordShah functional in image processing," in the Proceedings of IEEE Workshop on Variational and Level Set Methods in Computer Vision, pp. 161-168, Vancouver, BC, Canada, 2001.

[39] T. F. Chan and L. A. Vese, "An efficient variational multiphase motion for the Mumford-Shah segmentation model," in the Proceedings of Thirty-Fourth Asilomar Conference on Signals, Systems and Computers, pp. 490-494, Pacific Grove, CA, USA, 2000.

[40] T. F. Chan and L. A. Vese, "Active contours without edges," IEEE Transactions on Image Processing, vol. 10, pp. 266 - 277, 2001.

[41] L. A. Vese and T. F. Chan, "A multiphase level set framework for image segmentation using the Mumford and Shah model," University of California, Los Angeles, CA, USA, Computational and Applied Mathematics Report 01-25, 2001.

[42] A. Tsai, A. Yezzi-Jr, and A. S. Willsky, "Curve evolution implementation of the Mumford-Shah functional for image segmentation, denoising, interpolation, and magnification," IEEE Transactions on Image Processing, vol. 10, pp. 1169 1186, 2001. 
[43] E. D. Angelini, J. Holmes, A. F. Laine, and S. Homma, "Segmentation of realtime 3D cardiac ultrasound with implicit deformable models without gradients," in the Proceedings of International Symposium on Image and Signal Processing and Analysis, pp. 711-716, Rome, Italy, 2003.

[44] N. Lin, W. Yu, and J. S. Duncan, "Combinative multi-scale level set framework for echocardiographic image segmentation," Medical Image Analysis, vol. 7, pp. 529-537, 2003.

[45] T. F. Chan, B. Y. Sandberg, and L. A. Vese, "Active contours without edges for vector-valued images," Journal of Visual Communication and Image Representation, vol. 11, pp. 130-141, 2000.

[46] J. B. Maintz and M. A. Viergever, "A survey of medical image registration," Medical Image Analysis, vol. 2, pp. 1-36, 1998.

[47] B. v. Ginneken, A. F. Frangi, J. J. Staal, B. M. t. H. Romeny, and M. A. Viergever, "Active shape model segmentation with optimal features," IEEE Transactions on Medical Imaging, vol. 21, pp. 924 -933, 2002.

[48] A. F. Frangi, D. Rueckert, J. A. Schnabel, and W. J. Niessen, "Automatic construction of multiple-object three-dimensional statistical shape models: application to cardiac modeling," IEEE Transactions on Medical Imaging, vol. 21, pp. $1151-1166,2002$.

[49] C. J. Taylor, T. F. Cootes, A. Hill, and J. Haslam, "Medical image segmentation using active shape models," in Medical Imaging, M. H. K. L. Beolchi, Ed. Amsterdam: IOS Press, 1994, pp. 121-144.

[50] E. D. Angelini and E. J. Ciaccio, "Optimized region finding and edge detection of knee cartilage surfaces from magnetic resonance images," Annals of Biomedical Engineering, vol. 31, pp. 336-345, 2003.

[51] Medical Image Registration: CRC Press, 2001.

[52] B. C. Vemuri, J. Ye, Y. Chen, and C. M. Leonard, "Image registration via levelset motion: Applications to atlas-based segmentation," Medical Image Analysis, vol. 7, pp. 1-20, 2003.

[53] H. M. Yahia, E. G. Huot, I. L. Herlin, and I. Cohen, "Geodesic distance evolution of surfaces: a new method for matching surfaces," in the Proceedings of IEEE CVPR, pp. 663-668, Hilton Head Island, SC, USA, 2000.

[54] I. Cohen and I. Herlin, "Curve matching using geodesic paths," in the Proceedings of IEEE CVPR, pp. 741-746, Santa Barbara, CA, USA, 1998.

[55] S. Lavallee and R. Szeliski, "Recovering the position and orientation of free-form objects from image contours using 3D distance maps," IEEE Transactions on Pattern Analysis and Machine Intelligence, vol. 17, pp. 378-390, 1995.

[56] M. Rousson and N. Paragios, "Shape priors for level set representations," in the Proceedings of European Conference on Computer Vision, pp. 78-93, Copenhagen, Denmark, 2002.

[57] N. Paragios, M. Rousson, and V. Ramesh, "Matching distance functions: A shapeto-area variational approach for global-to-local registration," in the Proceedings of European Conference in Computer Vision, pp., Copenhagen, Denmark, 2002.

[58] N. Paragios and M. Rousson, "Shape analysis towards model-based segmentation," in Geometric Level Set Methods in Imaging, Vision and Graphics, N. P. S. Osher, Ed. New York: Springer Verlag, 2003, pp. 231-250. 
[59] A. Yezzi, L. Zollei, and T. Kapur, "A variational framework for joint segmentation and registration," in the Proceedings of IEEE Workshop on Mathematical Methods in Biomedical Image Analysis, pp. 44-51, Kauai, HI, USA, 2001.

[60] B. Vemuri and Y. Chen, "Joint image registration and segmentation," in Geometric Level Set Methods in Imaging, Vision and Graphics, N. P. S. Osher, Ed. New York: Springer Verlag, 2003, pp. 251-269.

[61] A. Tsai, A. Yezzi-Jr., W. Wells, III, C. Tempany, D. Tucker, A. Fan, W. E. Grimson, and A. Willsky, "Model-based curve evolution technique for image segmentation," in the Proceedings of Conference on Computer Vision and Pattern Recognition, pp. 463-468, 2001.

[62] D. Cremers, C. Schnorr, and J. Weickert, "Diffusion-snakes: combining statistical shape knowledge and image information in a variational framework," in the Proceedings of Workshop on Variational and Level Set Methods in Computer Vision, pp. 137-144, Vancouver, BC, Canada, 2001.

[63] N. Paragios, "A level set approach for shape-driven segmentation and tracking of the left ventricle," IEEE Transactions on Medical Imaging, vol. 22, pp. 773 -776, 2003.

[64] Y. Wang and L. Staib, "Boundary finding with correspondence using statistical shape models," in the Proceedings of IEEE Conference on Computer Vision and Pattern Recognition, pp., Santa Barbara, CA, USA, 1998.

[65] L. H. Staib and J. S. Duncan, "Model-based deformable surface finding for medical images," IEEE Transactions on Medical Imaging, vol. 15, pp. 720-731, 1996.

[66] Y. Chen, S. Thiruvenkadam, H. D. Tagare, F. Huang, D. Wilson, and E. A. Geiser, "On the incorporation of shape priors into geometric active contours," in IEEE Workshop on Variational and Level Set Methods in Computer Vision. Vancouver, BC, Canada, 2001, pp. 145-152.

[67] Y. Chen, F. Huang, H. D. Tagare, R. Murali, D. Wilson, and E. A. Geiser, "Using prior shape and intensity profile in medical image segmentation," in the Proceedings of IEEE ICCV, pp. 1117-1124, 2003.

[68] Y. Chen, S. Thiruvenkadam, H. D. Tagare, F. Huang, D. Wilson, and E. A. Geiser, "On the incorporation of shape priors into geometric active contours," in the Proceedings of IEEE Workshop on Variational and Level Set Methods in Computer Vision, pp. 145-152, Vancouver, BC, Canada, 2001.

[69] N. Paragios, M. Rousson, and V. Ramesh, "Knowledge-based registration \& segmentation of the left ventricle: a level set approach," in the Proceedings of IEEE Workshop on Applications of Computer Vision, pp. 37-42, 2002.

[70] N. Paragios, "A variational approach for the segmentation of the left ventricle in MR cardiac images," in the Proceedings of IEEE Workshop on Variational and Level Set Methods in Computer Vision, pp. 153-160, Vancouver, BC, Canada, 2001.

[71] C. Xu and J. L. Prince, "Snakes, shapes and gradient vector Flow," IEEE Transactions on Image Processing, pp. 359-369, 1998. 
[72] X. Han, C. Xu, and J. Prince, "Topology preserving geometric deformable models for brain reconstruction," in Geometric Level Set Methods in Imaging, Vision and Graphics, N. P. S. Osher, Ed. New York: Springer Verlag, 2003, pp. 421-438.

[73] R. K.-S. Kwan, A. C. Evans, and G. B. Pike, "An extensible MRI simulator for post-processing evaluation," in the Proceedings of Visualization in Biomedical Computing, pp. 135-140, Hamburg, Germany, 1996.

[74] D. L. Collins, A. P. Zijdenbos, V. Kollokian, J. G. Sled, N. J. Kabani, C. J. Holmes, and A. C. Evans, "Design and construction of a realistic digital brain phantom," IEEE Transactions on Medical Imaging, vol. 17, pp. 463-468, 1998.

[75] C. A. Cocosco, V. Kollokian, R. K.-S. Kwan, A. C. Evans, and v., no.4, part 2/4, S425, 1997 -- Proceedings of „, May 1997., "BrainWeb: online interface to a 3d MRI simulated brain database," in the Proceedings of 3-rd International Conference on Functional Mapping of the Human Brain, pp. S425, Copenhagen, Denmark, 1997.

[76] R. K.-S. Kwan, A. C. Evans, and G. B. Pike, "MRI simulation-based evaluation of image-processing and classification methods," IEEE Transactions on Medical Imaging, vol. 18, pp. 1085-1097, 1999.

[77] J. Mykkanen, J. Tohka, and U. Ruotsalainen, "Automated delineation of brain stuctures with snakes in PET," in Physiological Imaging of the Brain with PET, A. Press, Ed., 2001, pp. 39-43.

[78] E. O. Ofili and N. C. Nanda, "Three-dimensional and four-dimensional echocardiography," Ultrasound Medical Biology, vol. 20, 1994.

[79] A. Fenster and D. B. Downey, "Three-Dimensional Ultrasound Imaging," in Handbook of Medical Imaging. Volume1. Physics and Psychophysics, vol. 1, H. L. K. Jacob Beutel, Richard L. Metter, Ed. Bellingham, WA, USA.: SPIE- The International Society of Optical Engineering, 2000, pp. 463-510.

[80] R. N. Rankin, A. Fenster, D. B. Downey, P. L. Munk, M. F. Levin, and A. D. Vellet, "Three-dimensional sonographic reconstruction: technique and diagnostic applications," American Journal of Radiology, vol. 161, pp. 695-702, 1993.

[81] M. Belohlavek, D. A. Foley, T. C. Gerber, T. M. Kinter, J. F. Greenleaf, and J. B. Seward, "Three- and four-dimensional cardiovascular ultrasound imaging: a new era for echocardiography," Mayo Clinic Proceedings, vol. 68, pp. 221-240, 1993.

[82] G. Stetten, T. Ota, C. Ohazama, C. Fleishman, J. Castelucci, J. Oxaal, T. Ryan, J. Kisslo, and O. T. Von Ramm, "Real-time 3D ultrasound: a new look at the heart," Journal of Cardiovascular Diagnosis Procedures, vol. 15, pp. 73-84, 1998.

[83] S. W. Smith, J. H. G. Pavy, J. A. Miglin, and O. T. Von Ramm, "Improved realtime volumetric ultrasonic imaging," Ultrasonic Imaging, vol. 14, pp. 186-211, 1992.

[84] O. T. Von Ramm, J. H. G. Pavy, S. W. Smith, and J. Kisslo, "Real-time, threedimensional echocardiography: the first human images," Circulation, vol. 84, pp. $685,1991$.

[85] O. T. Von Ramm and S. W. Smith, "Real time volumetric ultrasound imaging system," Journal of Digital Imaging, vol. 3, pp. 261-266, 1990.

[86] T. Ota, C. E. Fleishman, M. Strub, G. Stetten, C. J. Ohazama, O. T. Von Ramm, and J. Kisslo, "Real-time, three-dimensional echocardiography: Feasibility of 
dynamic right ventricular volume measurement with saline contrast.," American Heart Journal, vol. 137, pp. 958-66, 1999.

[87] J. Kisslo, B. Firek, T. Ota, D. H. Kang, C. E. Fleishman, G. Stetten, J. Li, C. J. Ohazama, D. Adams, C. Landolfo, T. Ryan, and O. T. Von Ramm, "Real-time volumetric echocardiography: the technology and the possibilities,"

Echocardiography, vol. 17, pp. 773-779, 2000.

[88] A. A. Morsy, G. D. Stetten, and O. T. Von Ramm, "Detection and quantification of true 3D motion components of the myocardium using 3D speckle tracking in volumetric ultrasound scans: simulations and initial experimental results," in the Proceedings of Medical Imaging 1997: Physiology and Function from Multidimensional Images, pp. 346-353, 1997.

[89] T. Ota, C. E. Fleishman, C. J. Ohazama, G. Stetten, C. W. Lewis, D. D. Glower, J. Li, T. Ryan, J. Kisslo, and O. T. Von Ramm, "Measurement of left ventricular volume by real-time, three-dimensional echocardiography in dogs," Circulation, vol. 94, pp. 379, 1990.

[90] D. P. Shattuck, M. D. Weinshenker, S. W. Smith, and O. T. Von Ramm, "Exploroscan: A parallel processing technique for high speed ultrasound imaging with linear phased arrays," The Journal of the Acoustical Society of America, vol. 75, pp. 1273-1282, 1984.

[91] T. Shiota, M. Jones, M. Chikada, C. Fleishman, J. Castellucci, B. Cotter, A. DeMaria, O. T. Von Ramm, J. Kisslo, T. Ryan, and D. Sahn, "Real-time three dimensional echocardiography for determining right ventricular stroke volume in an animal model of chronic right ventricular volume overload," Circulation, vol. 97, pp. 1897-1900, 1998.

[92] P. Masson and W. Pieczynski, "SEM algorithm and unsupervised statistical segmentation of satellite images," IEEE Transactions on Geoscience and Remote Sensing, vol. 31, pp. 618-633, 1993.

[93] M. B. I. Centre: Montréal Neurological Institute, McGill University, Montréal, Canada.

[94] Handbook of Medical Informatics. Heidelberg: Springer-Verlag, 2002.

[95] X. Han, C. Xu, M. Rettmann, and J. Prince, "Automatic segmentation editing for cortical surface reconstruction," in the Proceedings of SPIE Medical Imaging, pp. 194-203, San Diego, CA, USA, 2001.

[96] W. E. Lorensen and H. E. Cline, "Marching cubes: A high resolution 3D surface construction algorithm," Computer Graphics, vol. 21, pp. 163-169, 1987.

[97] S. Resnick, A. Goldszal, C. Davatzikos, S. Golski, M. Kraut, E. Metter, R. Bryan, and A. Zonderman, "One-year changes in MRI brain volumes in older adults," Cerebral Cortex, vol. 10, pp. 464-472, 2000.

[98] D. L. Pham and J. L. Prince, "Adaptive fuzzy segmentation of magnetic resonance images," IEEE Transactions on Medical Imaging, vol. 18, pp. 737-752, 1999.

[99] X. Han, C. Xu, U. Braga-Neto, and J. Prince, "Topology correction in brain cortex segmentation using a multiscale graph-based algorithm," IEEE Transactions on Medical Imaging, vol. 21, pp. 109-121, 2002.

[100] C. Xu, D. L. Pham, M. E. Rettmann, D. N. Yu, and J. L. Prince, "Reconstruction of the human cerebral cortex from magnetic resonance images," IEEE Transactions on Medical Imaging, vol. 18, pp. 467-480, 1999. 
[101] M. G. H. Center for Morphometric Analysis, "Brain Segmentation Repository."

[102] J. C. Rajapakse and F. Fruggel, "Segmentation of MR images with intensity inhomogeneities," Image and Vision Computing, vol. 16, pp. 165-180, 1998.

[103] R. T. Schultz and A. Chakraborty, "Magnetic resonance image analysis," in Handbook of Human Brain Function: Neuroimaging, E. Bigler, Ed. New York: Plenum, 1996.

[104] B. Pakkernenberg and H. J. Gundersen, "Neocortical neuron number in humans: effect of sex and age," Journal of Comparative Anatomy, vol. 384, 1997.

[105] C. Corsi, G. Saracino, A. Sarti, and C. Lamberti, "Left ventricular volume estimation for real-time three-dimensional echocardiography," IEEE Transactions on Medical Imaging, vol. 21, pp. 1202 -1208, 2002.

[106] E. Angelini, A. Laine, S. Takuma, J. Holmes, and S. Homma, "LV volume quantification via spatio-temporal analysis of real-time 3D echocardiography," IEEE Transactions on Medical Imaging, vol. 20, pp. 457-469, 2001.

[107] J. Udupa, V. LeBlanc, H. Schmidt, C. Imielinska, P. Saha, G. Grevera, Y. Zhuge, P. Molholt, Y. Jin, and L. Currie, "A methodology for evaluating image segmentation algorithm," in the Proceedings of SPIE Conference on Medical Imaging, pp. 266-277, San Diego CA, USA, 2002. 